$$
\begin{aligned}
& \text { تقصي الخصائص السيكومترية لاختبار ذكاء جمعي حسب نظرية السمات الكامنة } \\
& \text { تغريد حجازي* و أحمد الشريفين } \\
& \text { جامعة اليرموك، الأردن }
\end{aligned}
$$

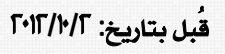

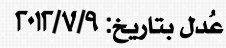

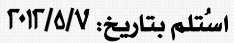

$$
\begin{aligned}
& \text { هدفت هذه الدراسة إلى تقصي الخصائص السيكومترية لاختبار المفردات بأسهائها واستعهالاتها ، وذلك حسب نظرية } \\
& \text { السهمات الكامنة. ولتحقيق ذلك تم تطبيق الإختبار على عينة مكونة من 7ع ب طالبا وطالبة بين سن 0- } 9 \text { سنوات، } \\
& \text { موزعين على الصفوف: التههيدي، الأول الأساسي، الثاني الأسـاسي، والثالث الأساسي، تم اختيارهم عشوائيا من } \\
& \text { المدارس الحكومية يوٌ مدينة إربد. استخدمت الدراسة النموذج الثنائي التدريج لتحليل الاستجابات. تكون الإختبار } \\
& \text { بصورته النهائية من } 17 \text { فقرة كانت مطابقة للنموذج، بوسط حسابي لمتوسطات المربعات الداخلية والخارجية يقترب من }
\end{aligned}
$$

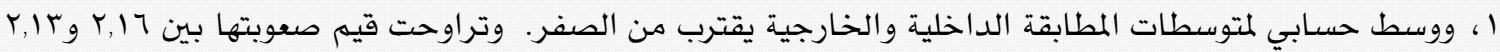

$$
\begin{aligned}
& \text { لوجيت وبهتوسط يساوي صفر لوجيت. وبلغت قيم معامل الثبات للأفراد والفقرات ای,.•، بو, · على التوالي. وتمتع } \\
& \text { المقياس بدلالات صدق متعددة. } \\
& \text { الكلمات المفتاحية: النموذج الثنائي التدريج، نظرية السهات الكامنة، معالم الفقرات، اختبار ذكاء. }
\end{aligned}
$$

\title{
Estimating Psychometric Characteristics of Collective Intelligence Test According to Latent Trait Theory
}

Taghreed Hijazi*\& Ahmad Alshreffen

Al Yarmouk University, Jordan

This Study Investigated the psychometric characteristics of vocabulary intelligence test according to latent trait theory, by administering the test to randomly selected 246 students between 5-9 years old distributed over Kg2 and 1st, 2nd and 3rd Primary grade from the government schools in Irbid city. The Dichotomous model was used to analyze data. The final form of the test consisted of 16 items fit the model with mean square infit/ outfit statistics about 1 , and standardized information weighted fit statistics about zero. Item difficulty parameters ranged from -2.16 to 2.13 logit with a mean of zero logit. The values of person reliability and item reliability were $0.81,0.92$ respectively. The test has multiple validity indicators.

Keywords: dichotomous model, latent trait theory, item parameter, intelligence test.

*taghreedah@yahoo.com 
ويعود البحـث في الفروق الفـردية في الذكاء إلى تاريخ

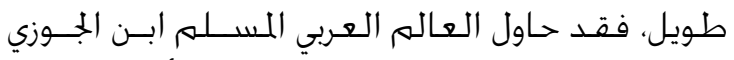

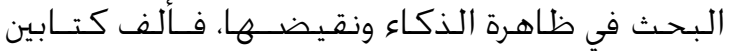

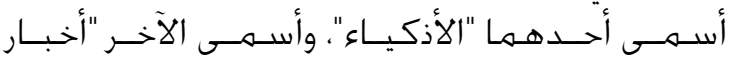

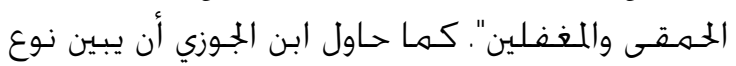

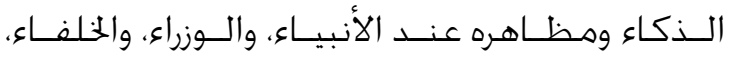

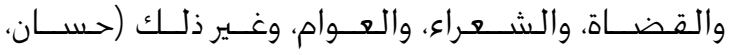

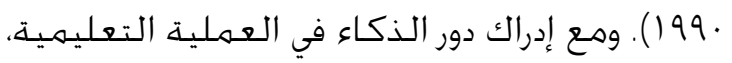

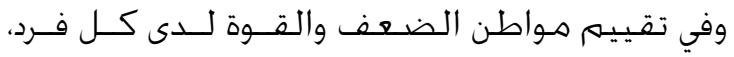

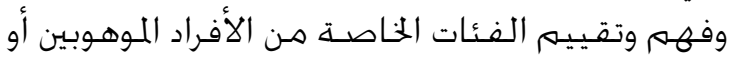

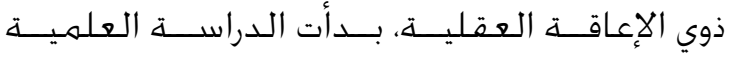

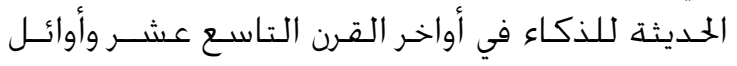

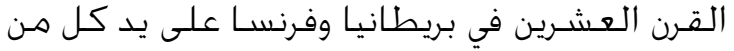

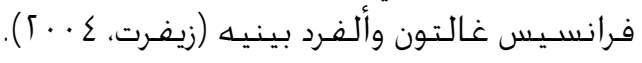

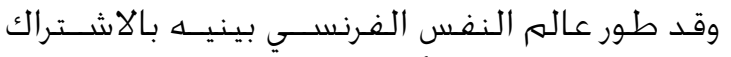

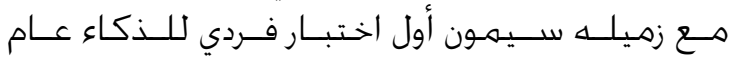

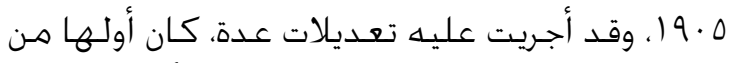

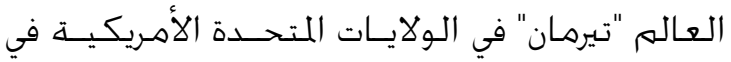

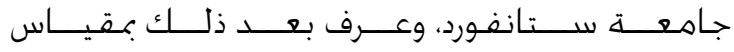

سـانفورد - بينيه (Anastasi \& Urbina, 1997).

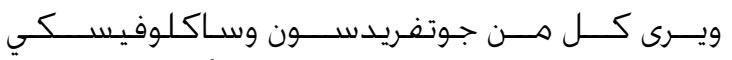
(Gottfredson \& Saklofske, 2009)

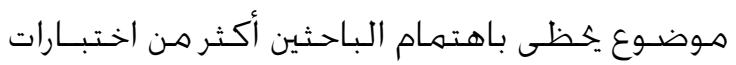

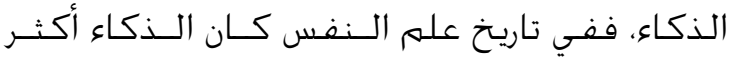

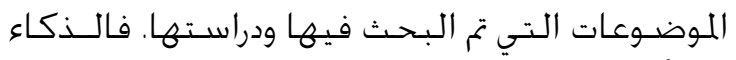

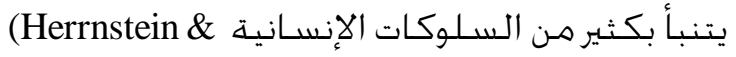
ويؤثر في كثير من جوانب الحياة مثل (Hurray, 1994) الشـخصـية (Eysenck, 1997)، والدافعية (Collins) (Ackerman \& و Messick, 2001) Kanfer, 2004)

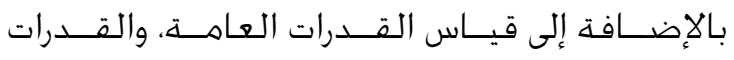

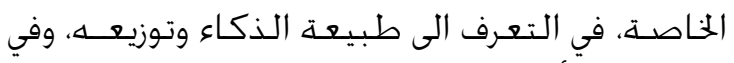

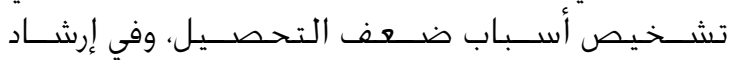

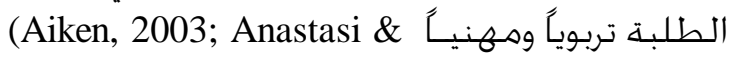
.Urbina, 1997)

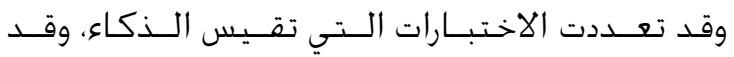

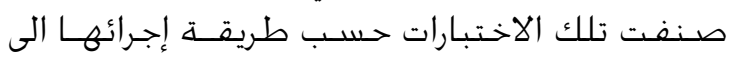

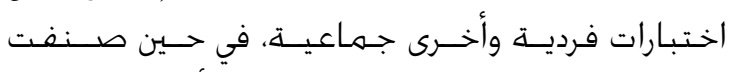

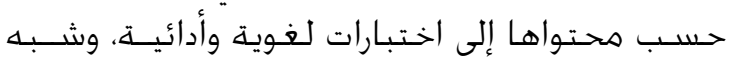

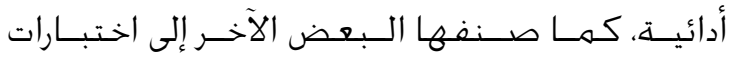

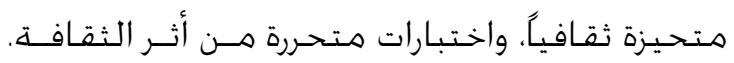

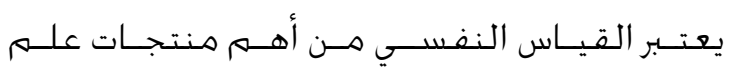

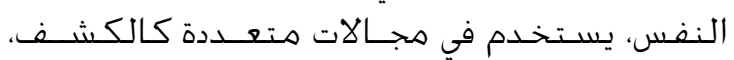

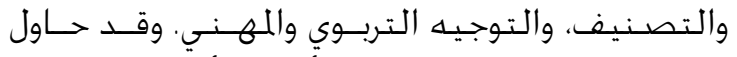

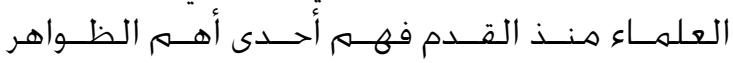

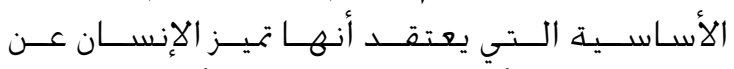

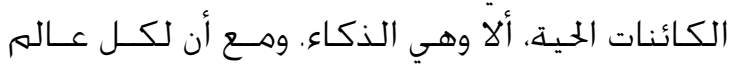

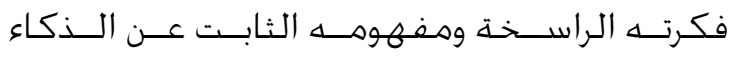

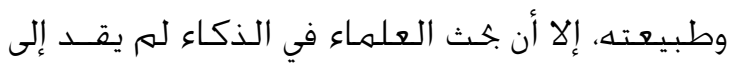

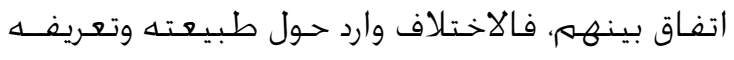

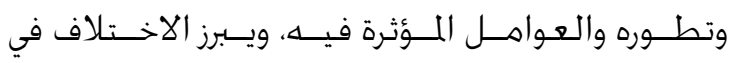

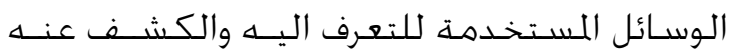

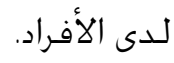

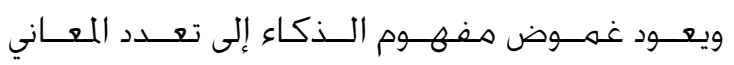

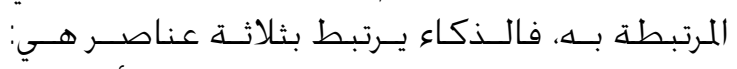

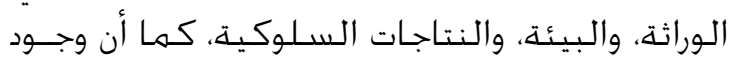

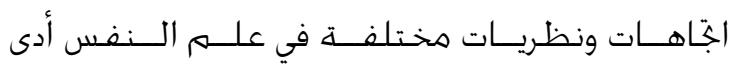

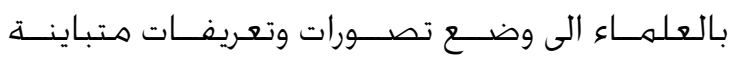

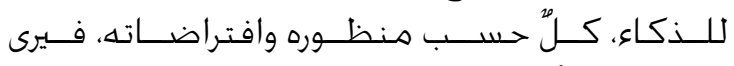

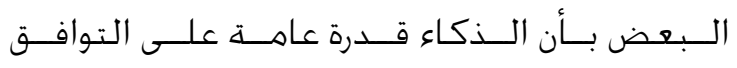

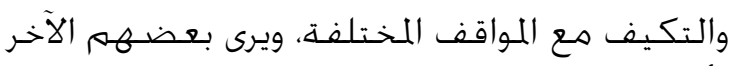

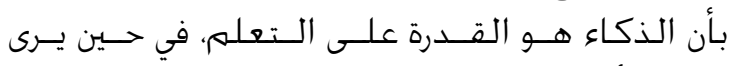

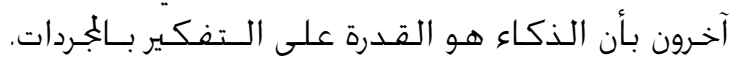

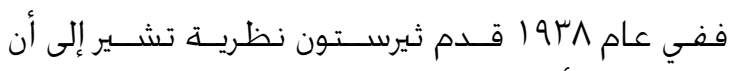

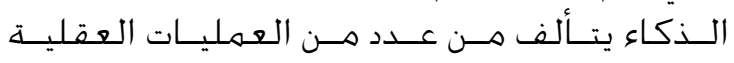

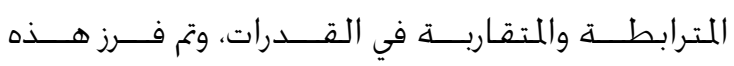

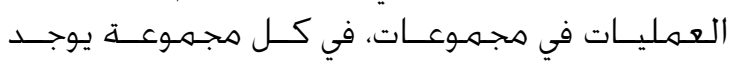

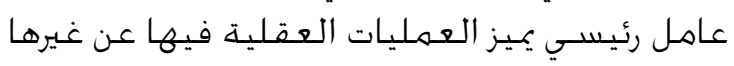

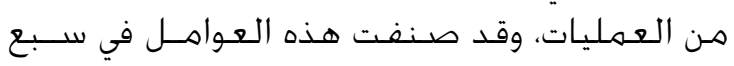

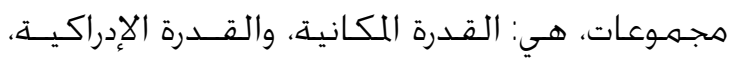

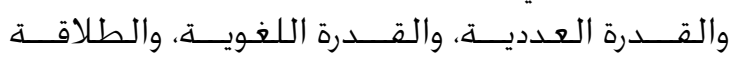

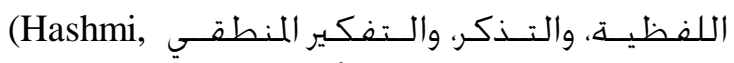
(Tirmizi, \& Shah; 2010)

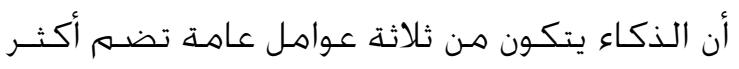

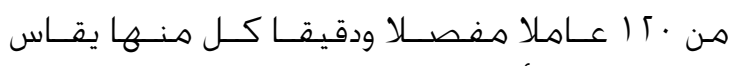

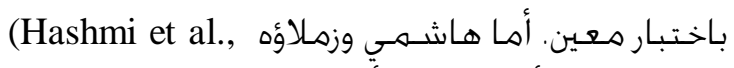

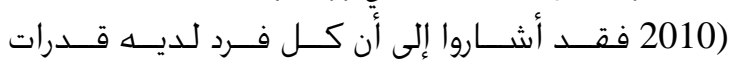

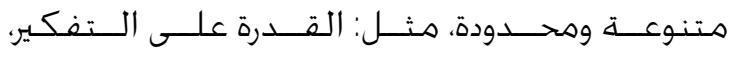

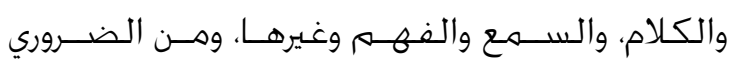

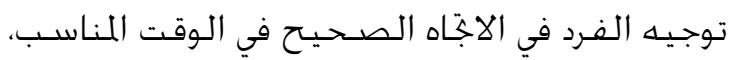
وفي الطريق الصــيح. 


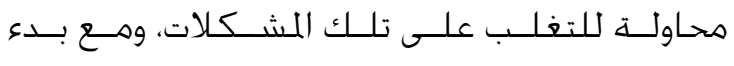

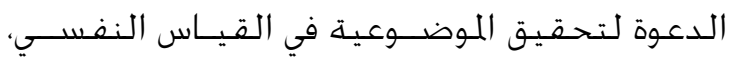

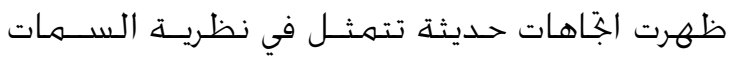

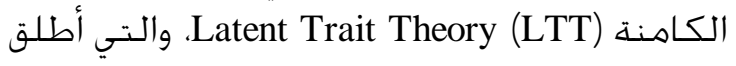

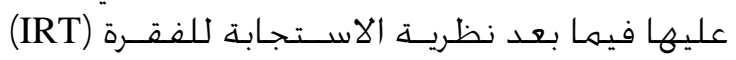
Item Response Theory يلاحظ ما تقدم أن سـمـة الذكاء قـد حظيت باهتمام

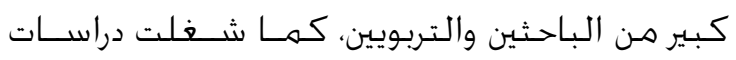

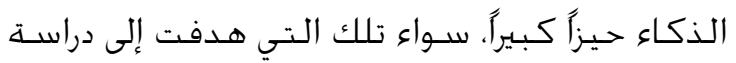

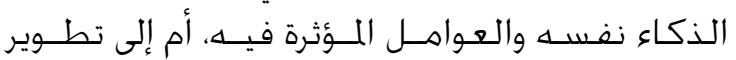

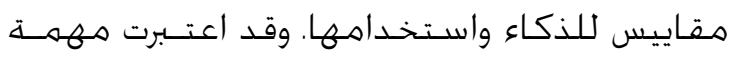

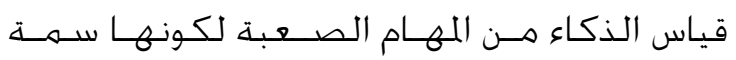

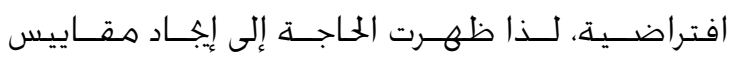

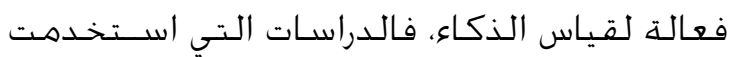

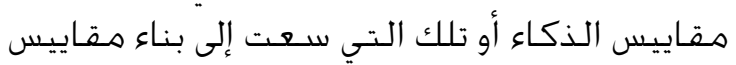

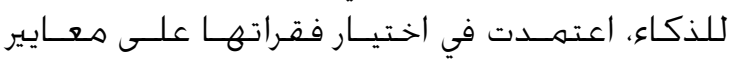

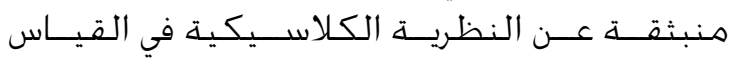

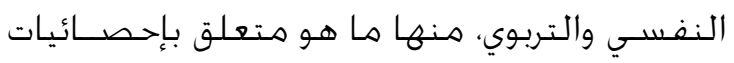

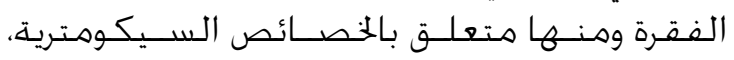

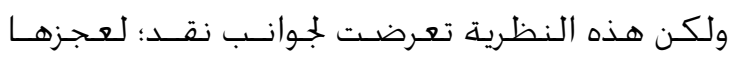

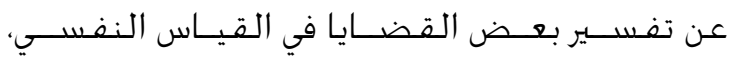

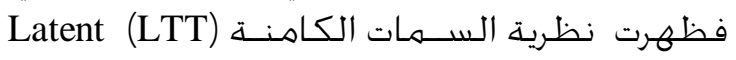

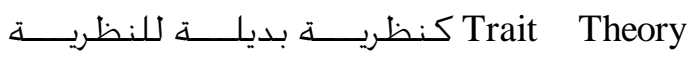

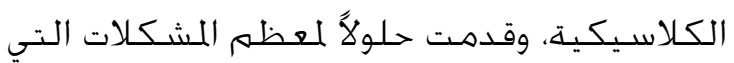

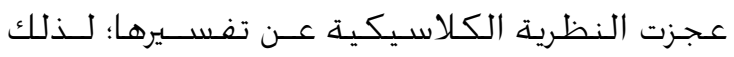

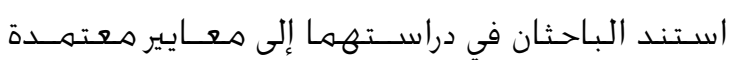

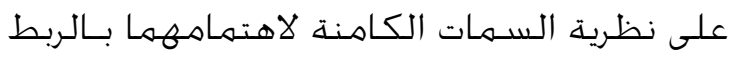

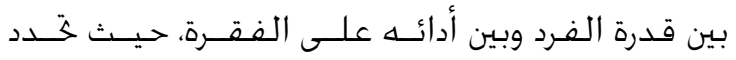

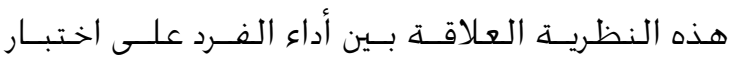

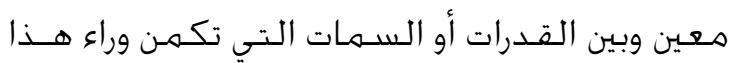

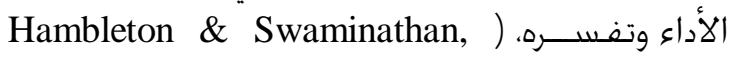

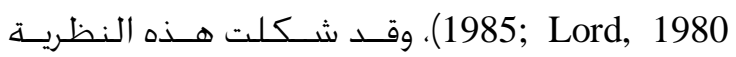

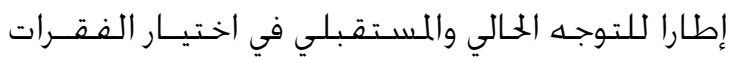

(Anastasi, 1982)

وتقوم هذه النظرية على مجهموعة من الافتراضـات

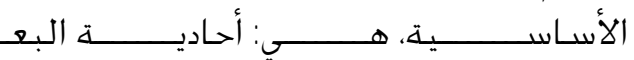

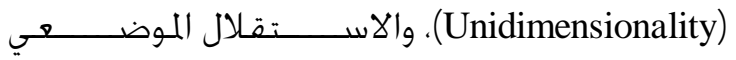
(Local Independence) الفقــرة (Item Characteristic Curve) فيعتـبر

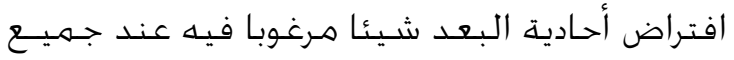

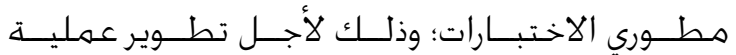

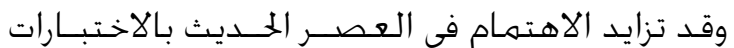

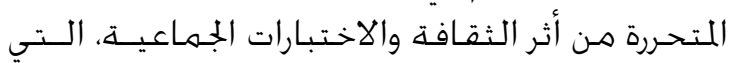

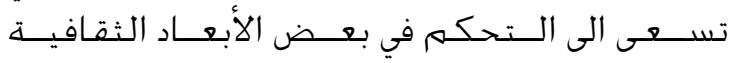
كالاختـاف اللغوي (أبو جـراد، ^ · · ؟).

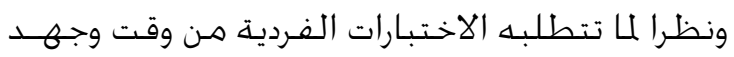

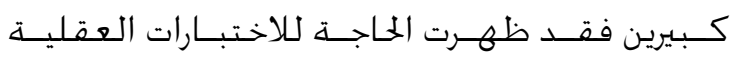

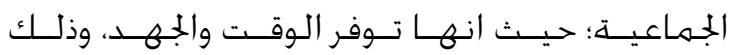

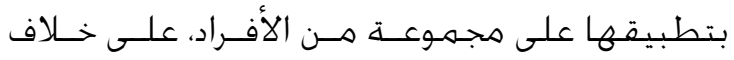

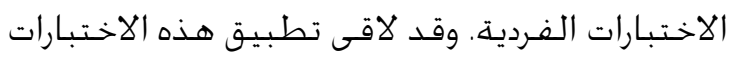

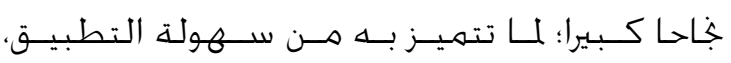

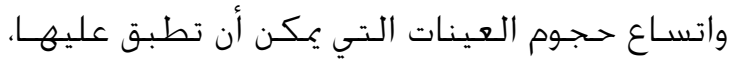

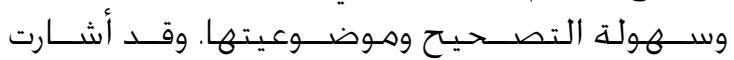
الدراسـات (Cronbach, 1960) إلى كفـاءة الاختبـــارات

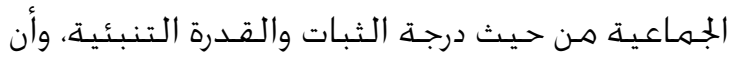

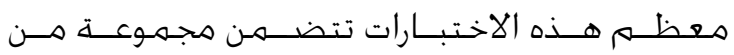

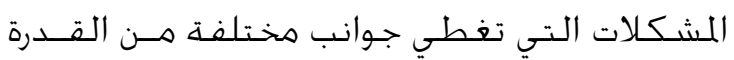

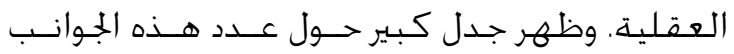

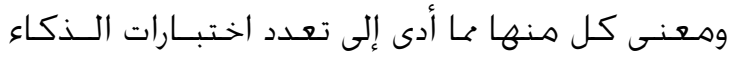

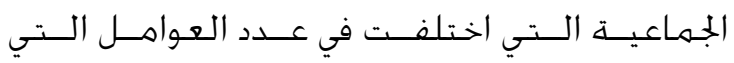

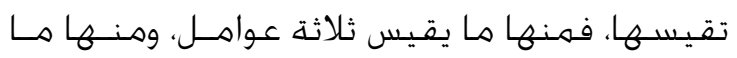

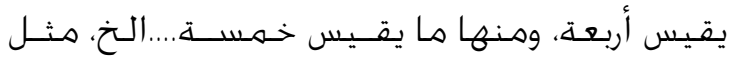

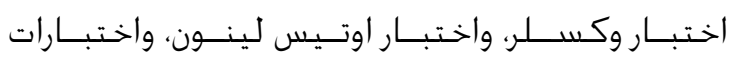

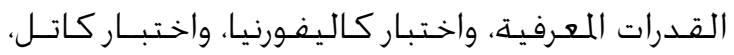

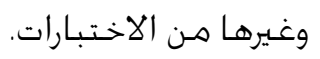

ويشـير عطوف (1911) أن اختبارات الذكاء تـؤدي دورا

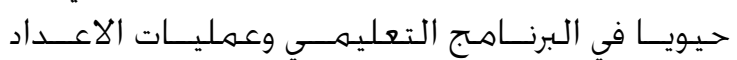

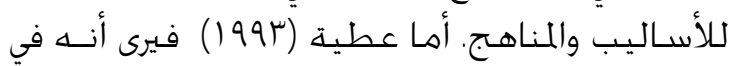

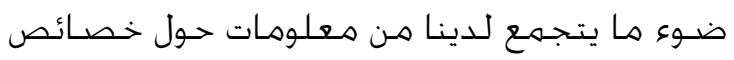

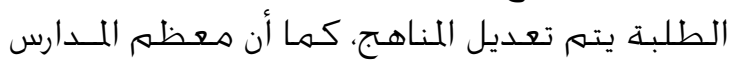

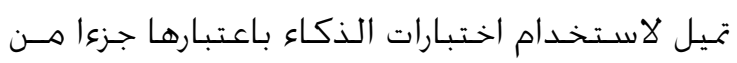

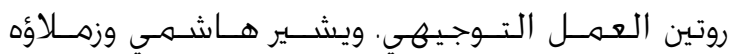

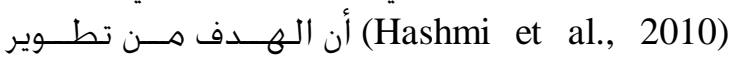

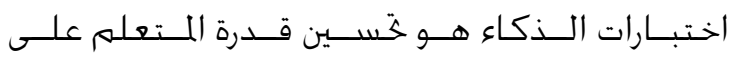

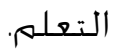

وقد وجهت العـديد من الإنتقادات لاختبارات الـــكاء الـاء

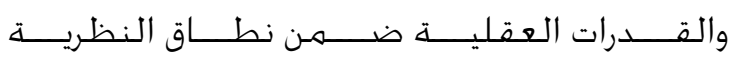

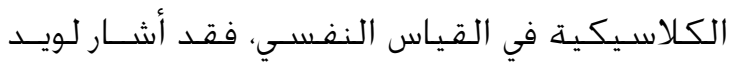

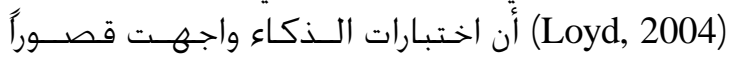

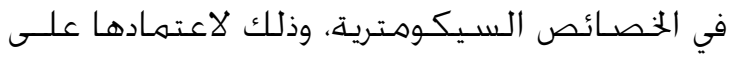

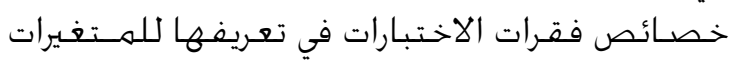

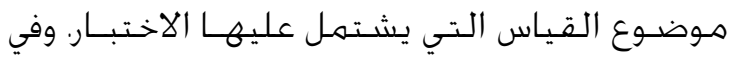




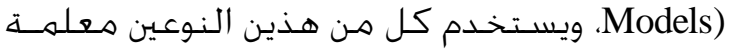

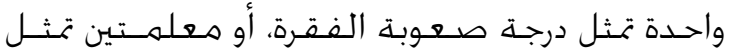

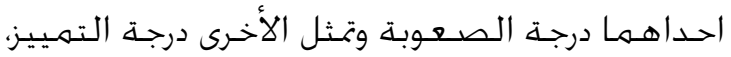

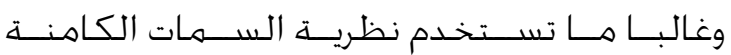

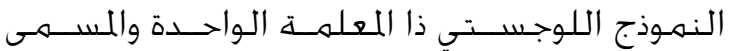
بنموذج راش (Rasch Model)؛ وذلك لما يتعيز به مــن النه

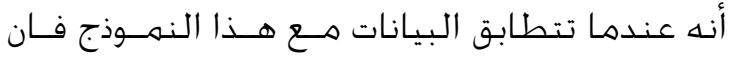

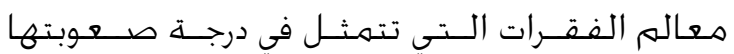

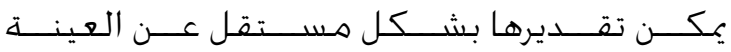

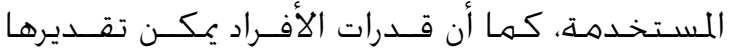

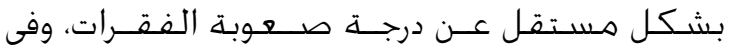

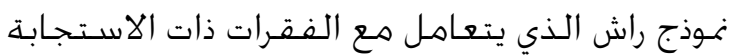

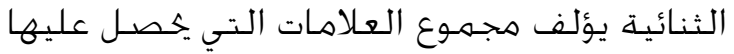

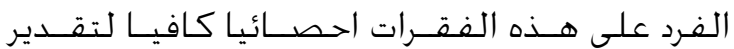

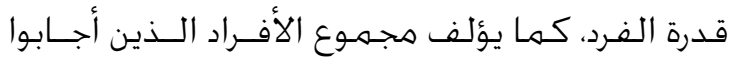

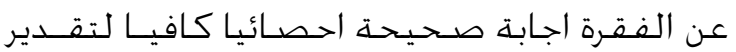

درجة صعوبتها (Masters, 1982).

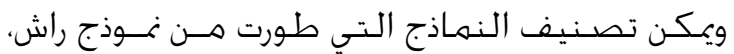

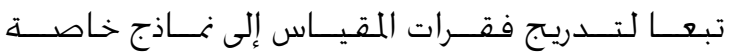

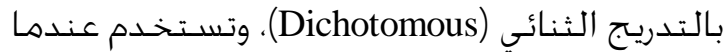

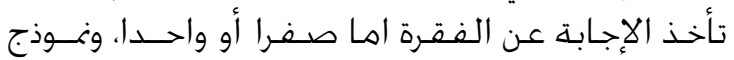

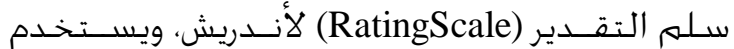

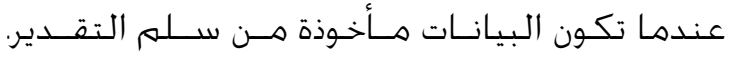

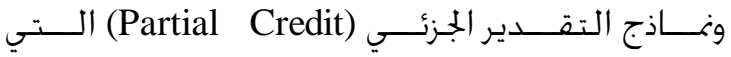

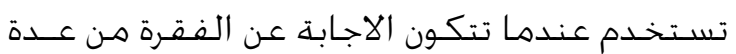

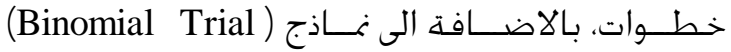

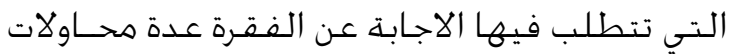

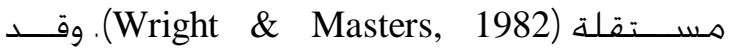

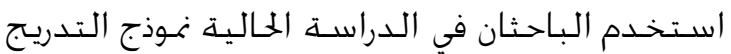

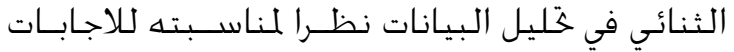

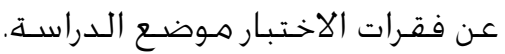

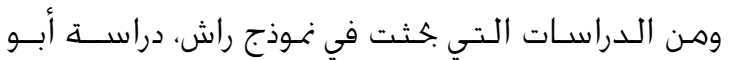

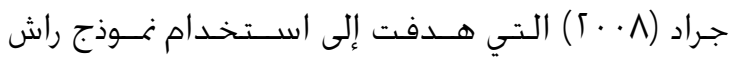

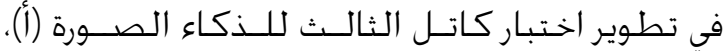

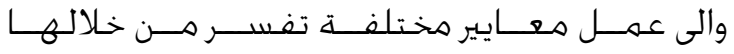

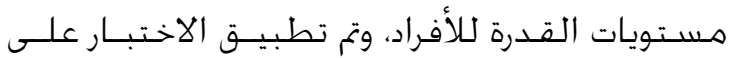

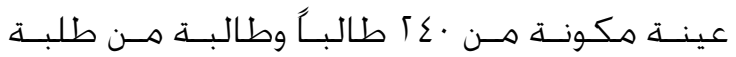

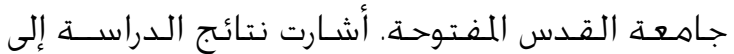

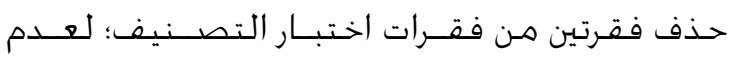

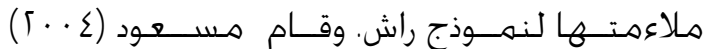

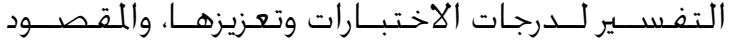

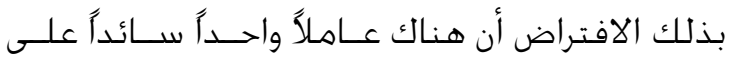

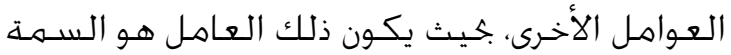

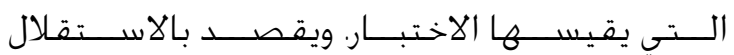

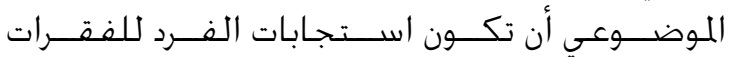

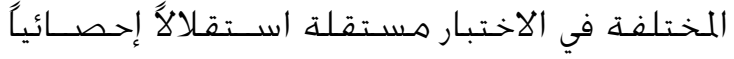

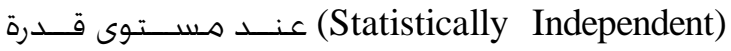

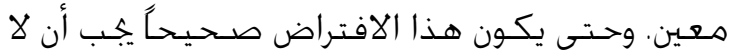

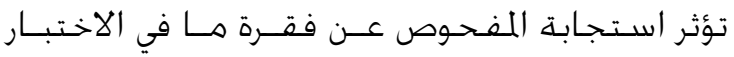

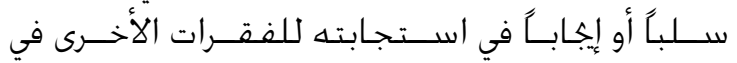

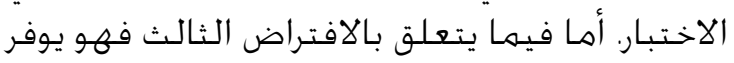

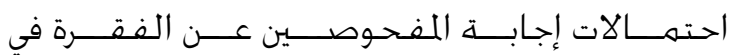

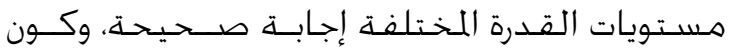

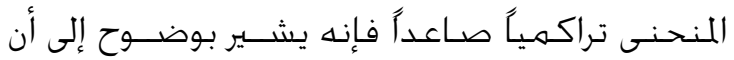

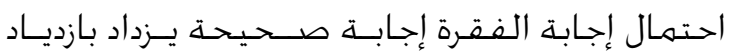

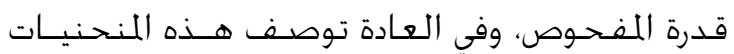

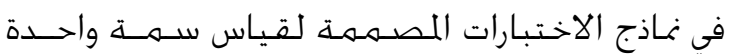

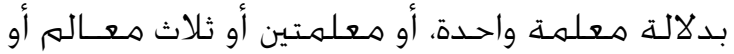

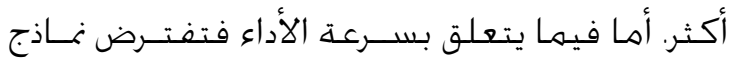

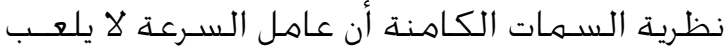

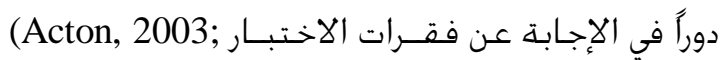
Albert \& Ghosh, 2000; Hambleton \& .Swaminathan, 1985)

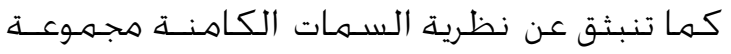

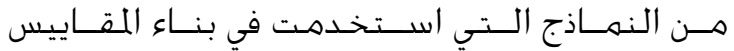

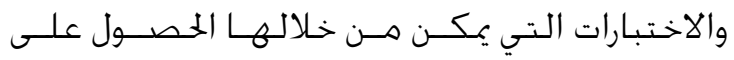

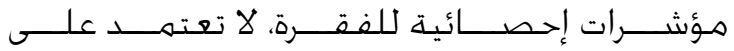

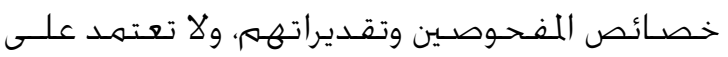

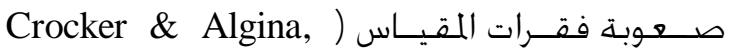

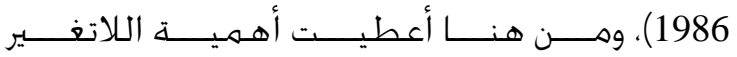

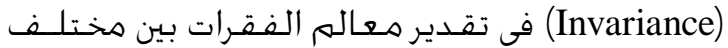

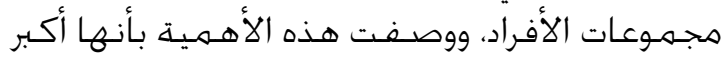

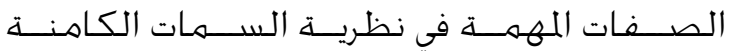
.(Lord, 1980)

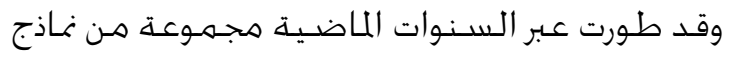

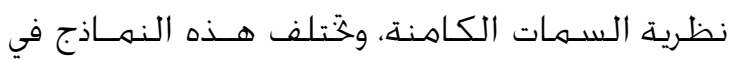

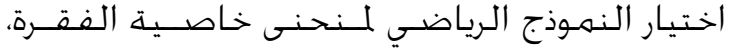

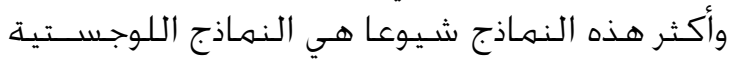

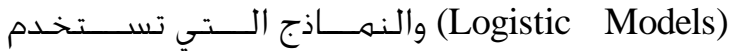

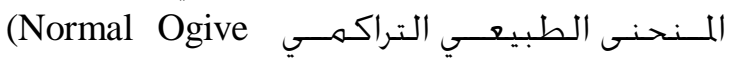




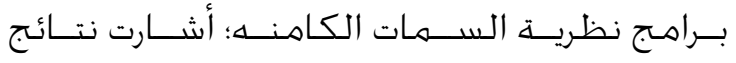

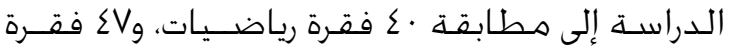
في القـراءة للنـموذج.

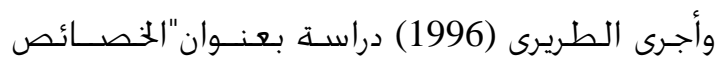

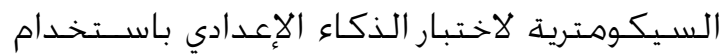

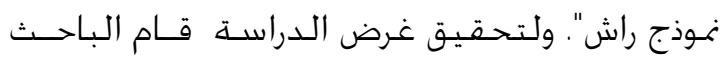

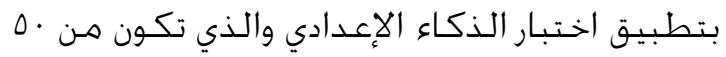

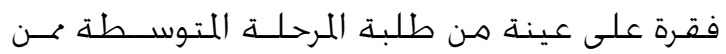

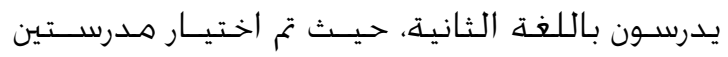

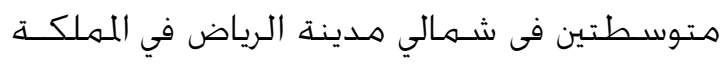

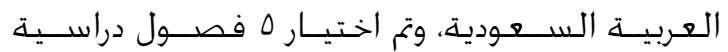

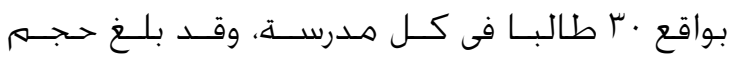

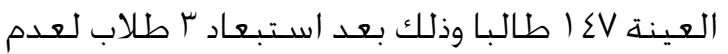

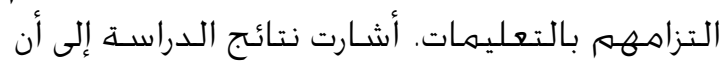

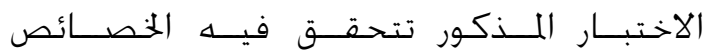

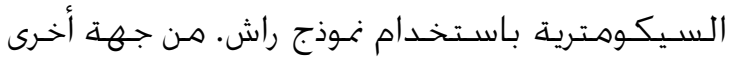

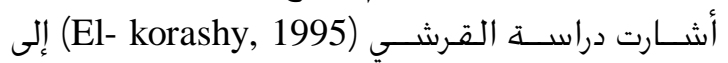

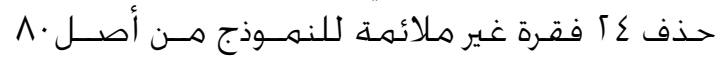

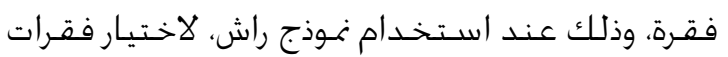

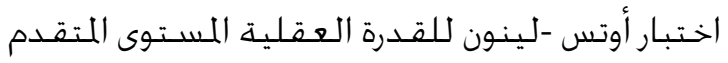

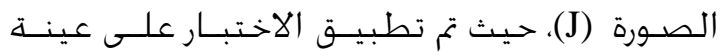

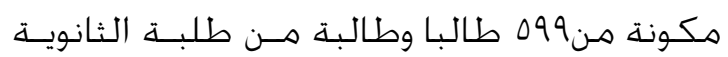

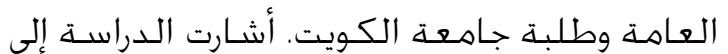

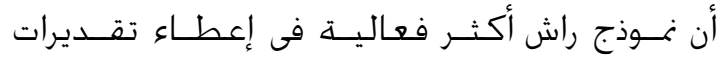

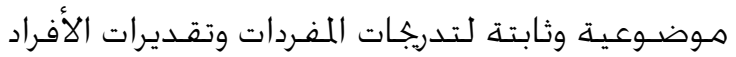

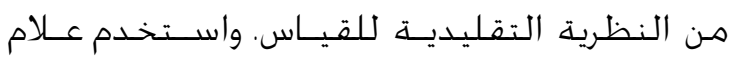

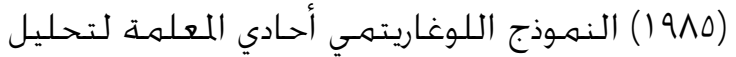

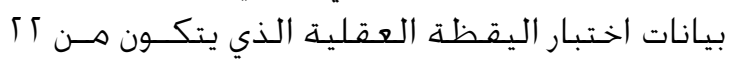

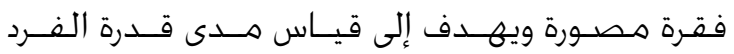

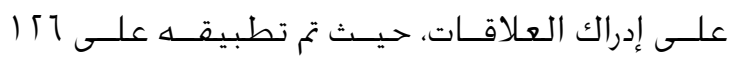

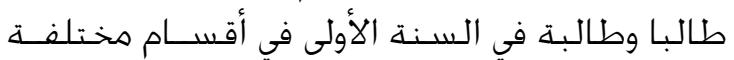

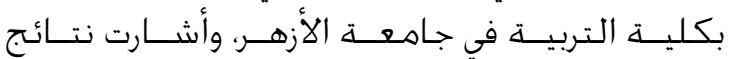

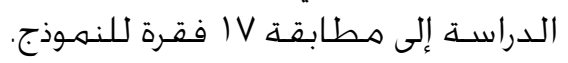

\section{مشكلة الدراسـة وأسئلتها}

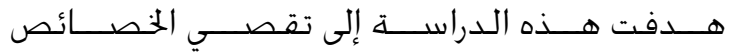

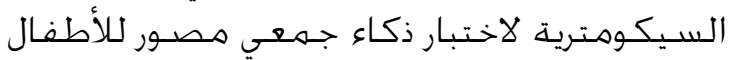

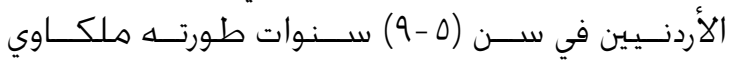

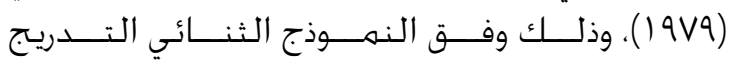

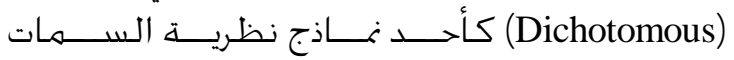

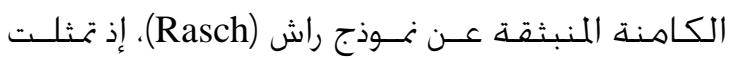

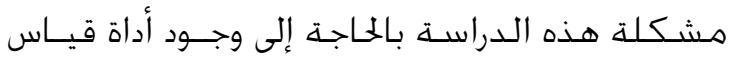

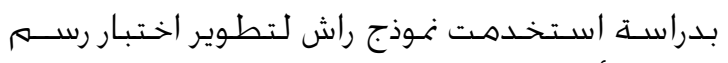

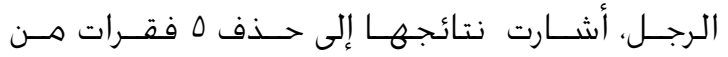

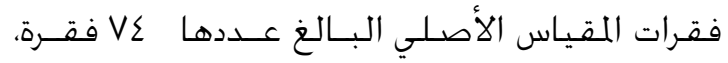

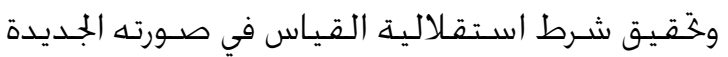

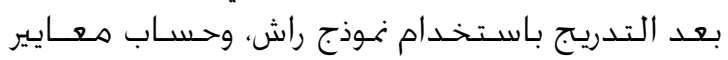

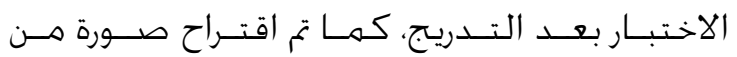

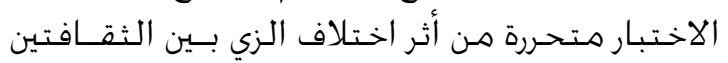

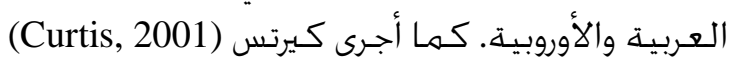

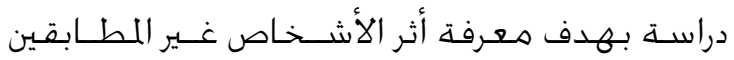

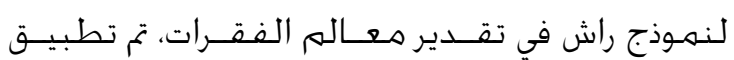

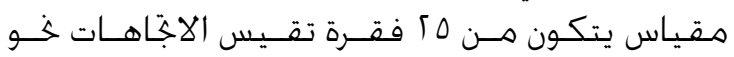

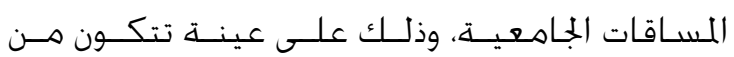

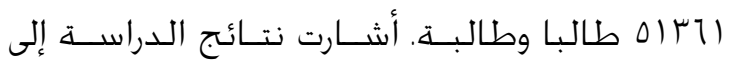

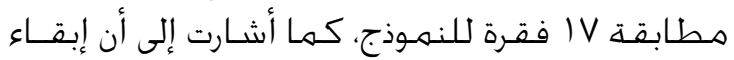

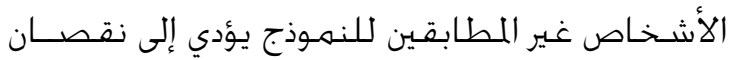

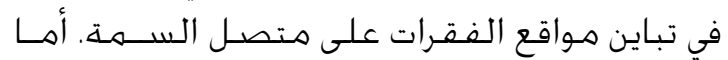

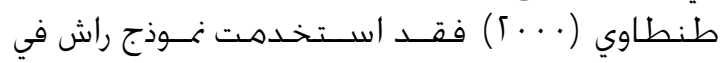

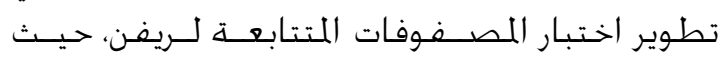

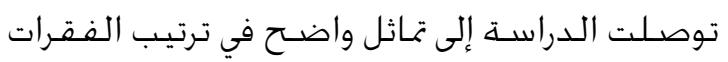

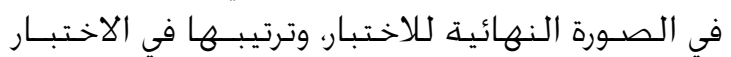

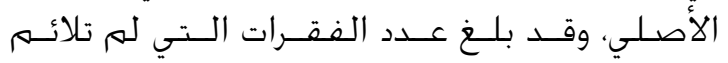

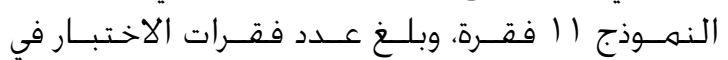

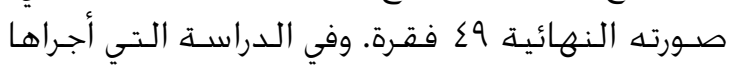

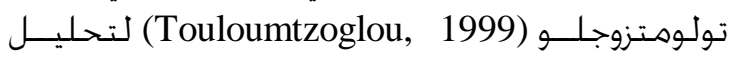

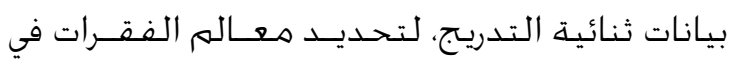

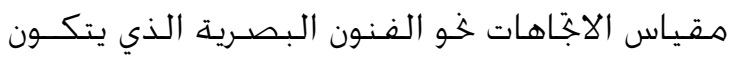

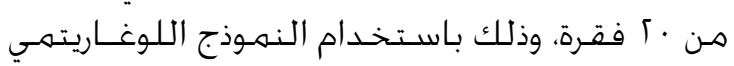

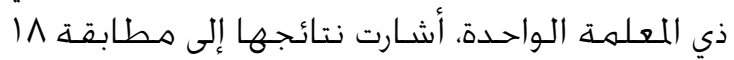

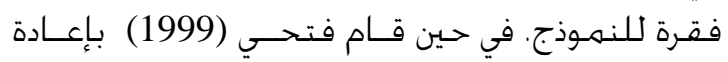

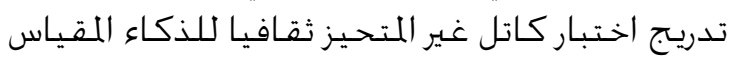

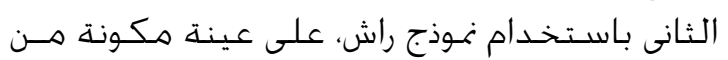

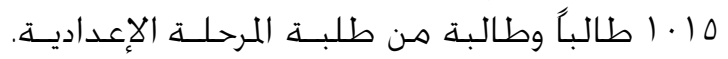

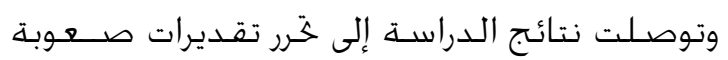

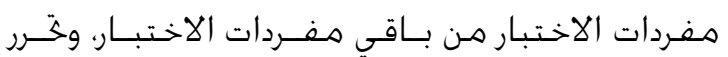

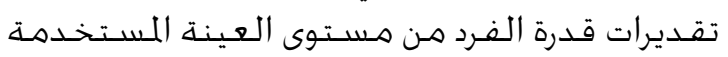

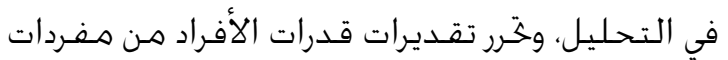

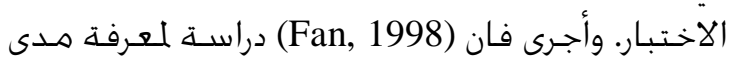

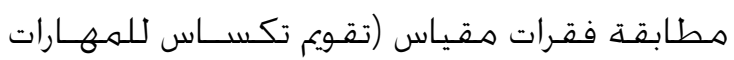

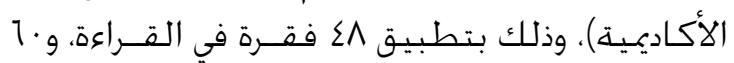

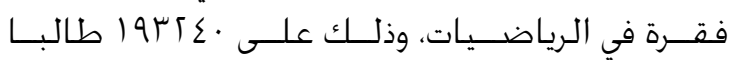

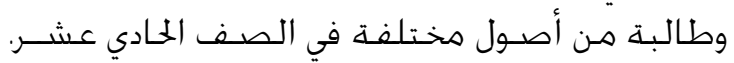

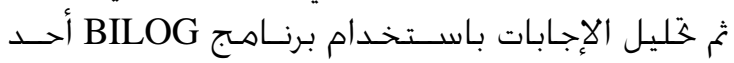




\section{محددات الدراســـ}

- اقتصـرت عينة الدراسـة على طلبة المدارس

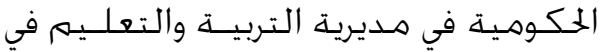

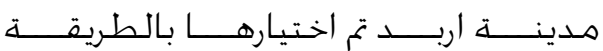

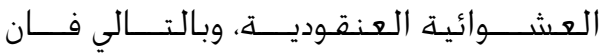

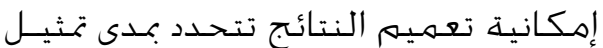

هذه العينة لطلبة المدارس.

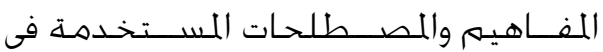

هذه الدراسـة محددة بالتعريفات الإجرائية،

وبالتالي فان إمكانية تعميم النتائج تتحدد الإنهائ

في ضـوء هذه التعريفات.

مصطلحات الدراسـة

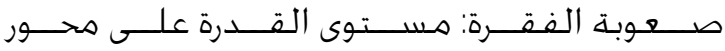

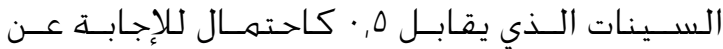

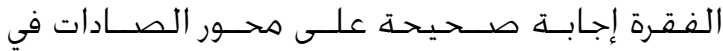

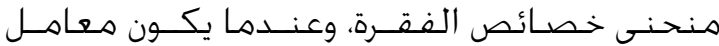
التخـمين يسـاوي صـفـرا.

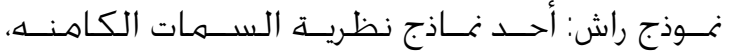

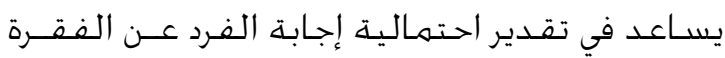

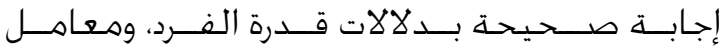

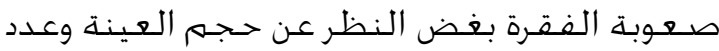

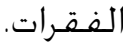

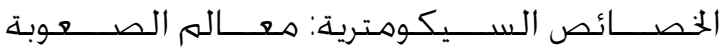
والصـــق والثبات لاختبار الذكاء التهاء

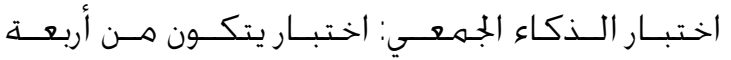

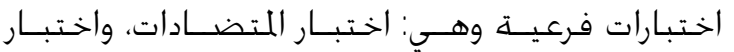

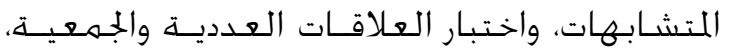

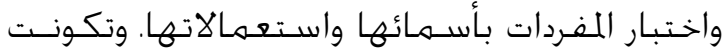

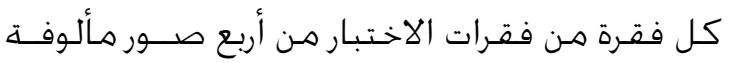
في البيئة الأردنية.

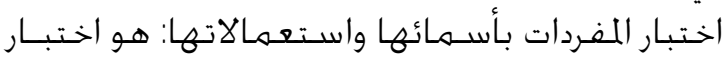

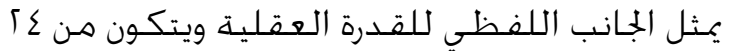
فقرة.

\section{الطريقـة والإجراءات}

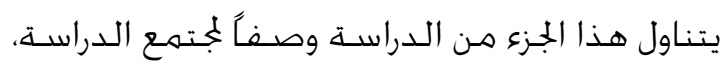

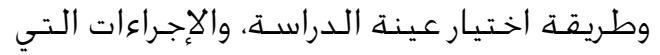

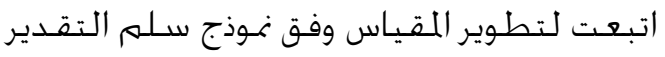
وهي على النحـو الآتي:

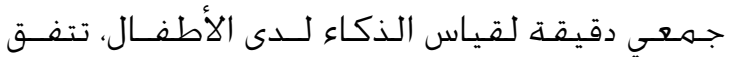

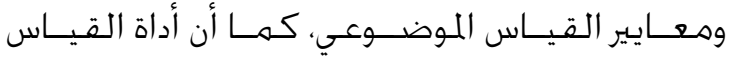

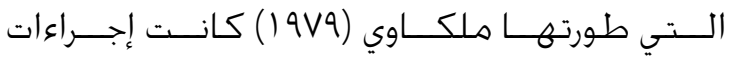

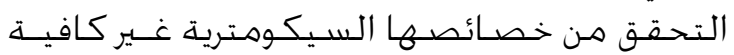

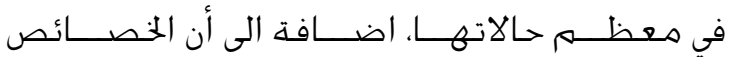

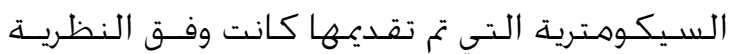

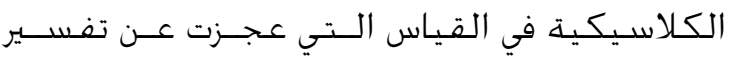

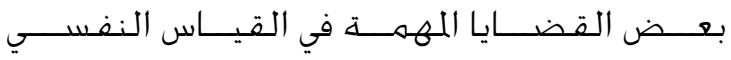

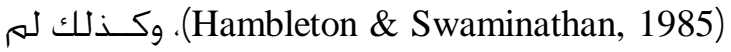
خُظ نظرية السـمات الكامنة باهتمام كبير وخاصـة

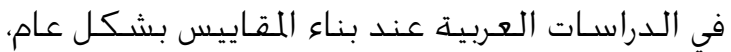

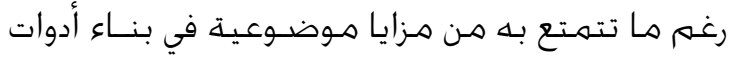

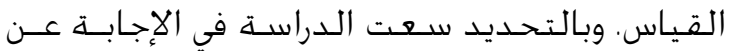
السـؤالين الاتيين:

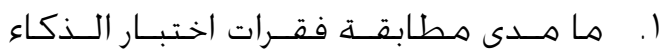

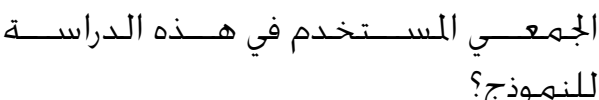
ז. ما الخصـائص السيكومترية لاختبار الذكاء لاءعاء

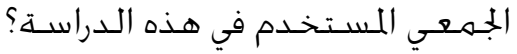

أهمية الدراسـة أنسئ

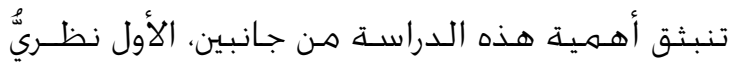

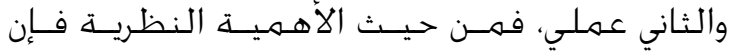

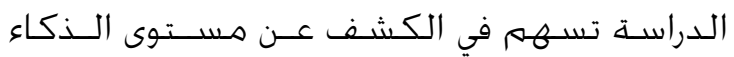

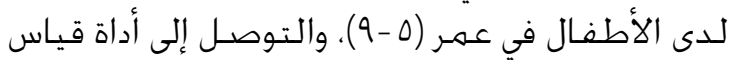

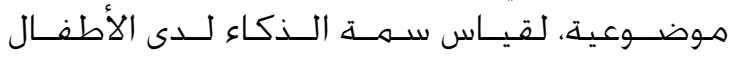

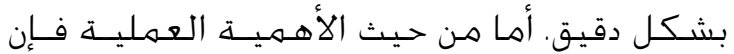

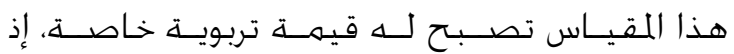

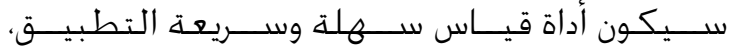

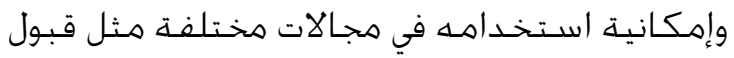

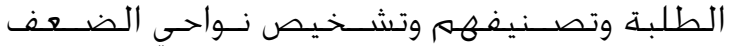

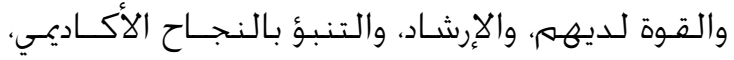

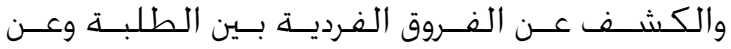

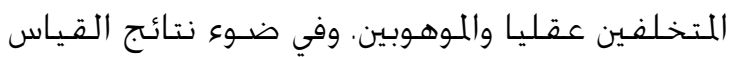

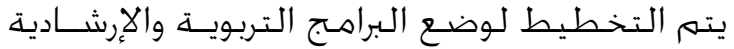

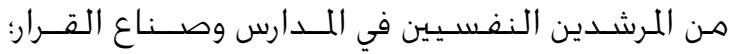

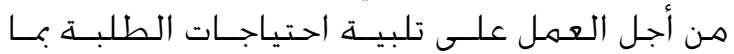

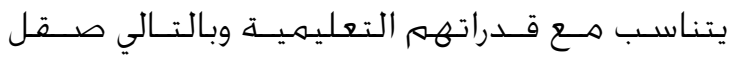
شـخصياتهم. 
الفقرات باستثنـاء الفقرتين لَ، ؟ )، وذلك حسـب ما

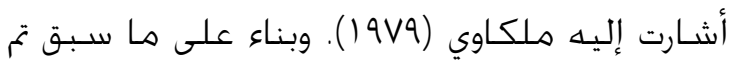

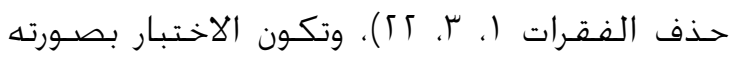

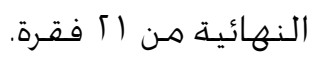

\section{النتائج ومناقشـنها}

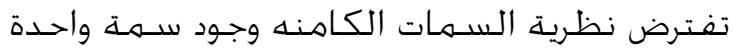

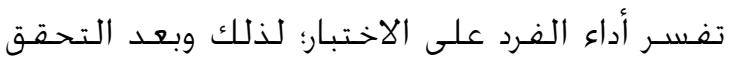

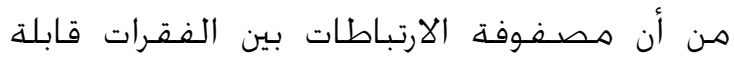

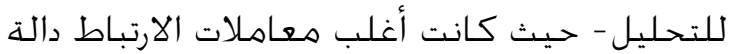

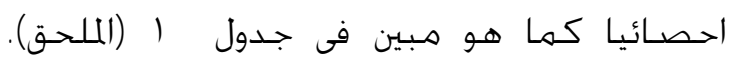

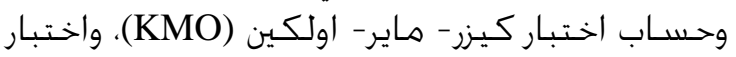

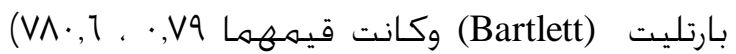

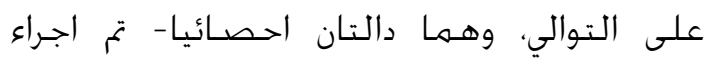

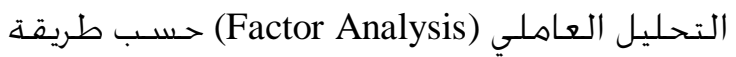

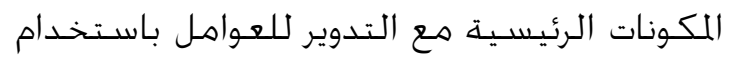

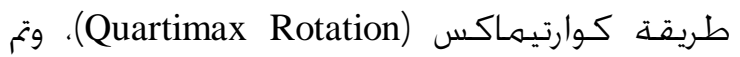

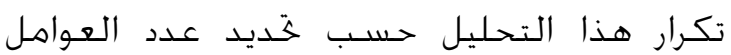

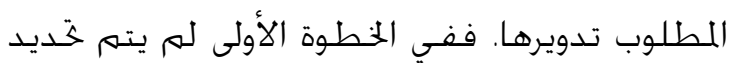

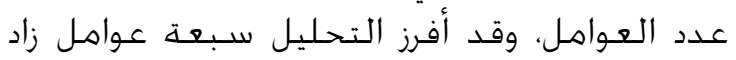

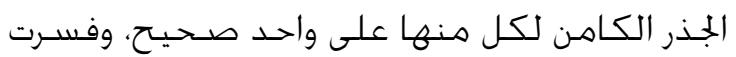

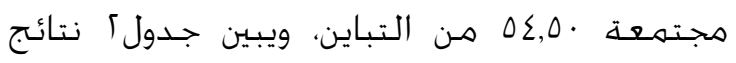

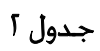

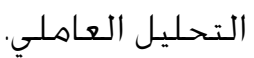

البناء العاملي، والجذر الكامن، ونسبة التباين لكل عامل،

ونسبة التباين التراكمية مرتبة تنازلياً

\begin{tabular}{|c|c|c|c|}
\hline المفسـر التراكعيـي & نسبة التباين & الكامن & العامل \\
\hline$\Gamma \cdot, \cdot 0$ & {$[\cdot, \cdot 0$} & $\varepsilon, \Gamma 1$ & 1 \\
\hline$[V, 1)$ & $\mathrm{V}, \cdot \mathrm{T}$ & I, ¿^ & r \\
\hline ד ז, & $1, \Gamma 0$ & $1, \Gamma 1$ & $r$ \\
\hline$r q .0$ & $0, \mathrm{~V}$. & I, r. & $\varepsilon$ \\
\hline$\sum \varepsilon, \Sigma r$ & $\Delta, r \wedge$ & $1,1 \%$ & 0 \\
\hline$\{9,0)$ & $0, \cdot \Lambda$ & $1, \cdot V$ & 1 \\
\hline$\Delta \varepsilon, 0$. & $\sum, 99$ & $1, \cdot 0$ & V \\
\hline
\end{tabular}

يتضح من جـدول ؟ أن العامل الأول يفسـر ما

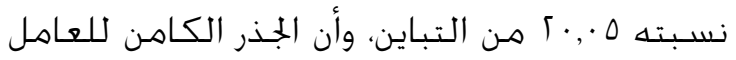

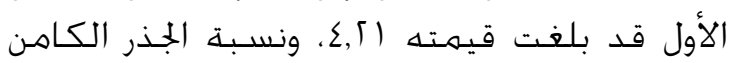

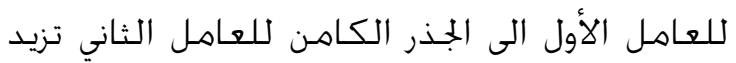

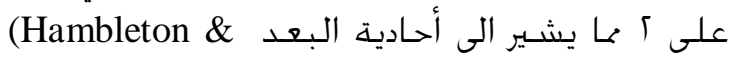

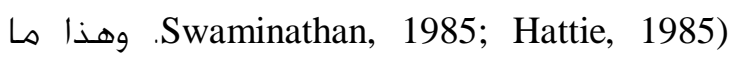

$$
\text { يؤكده منحـنى المنحـدر في شكل ا. }
$$

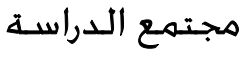

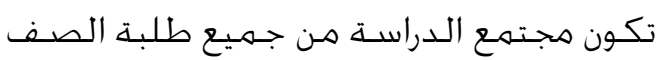

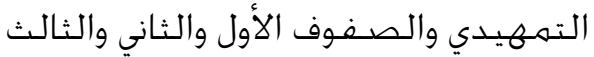

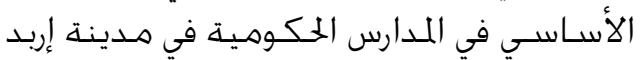

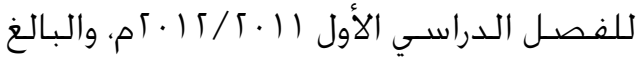

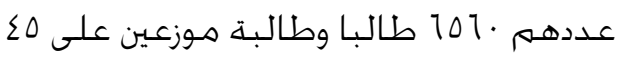
مدرسـة. عينة الدراسـة

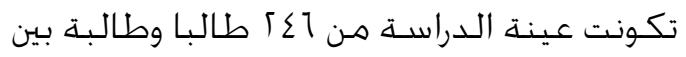

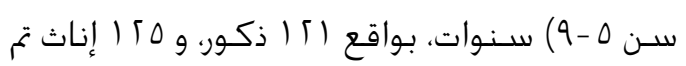

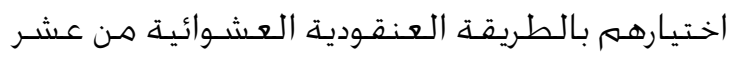

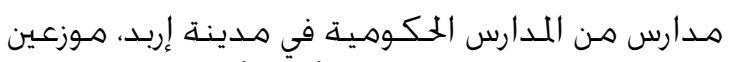

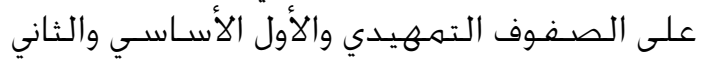

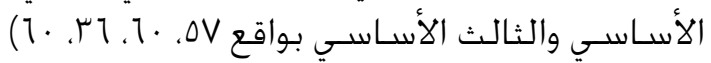

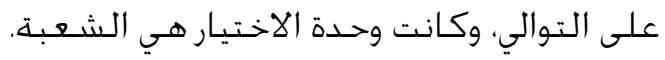

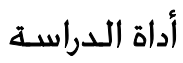

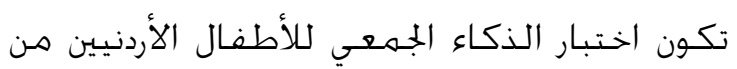

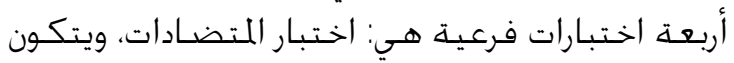

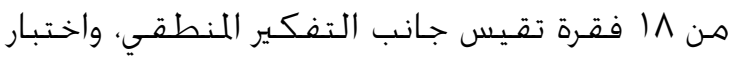

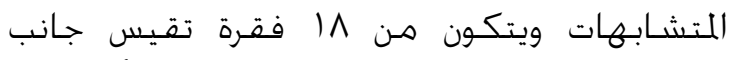
التفكير المنطقي، واختبار المفردات بأسـمائها

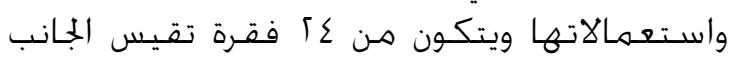

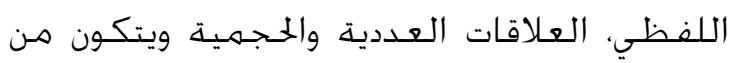

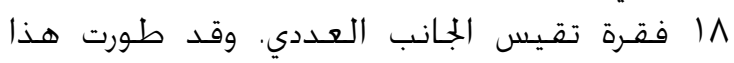

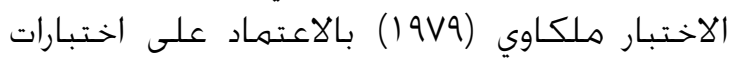

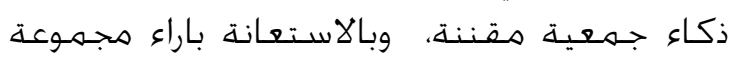

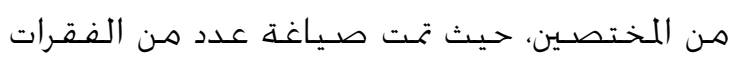

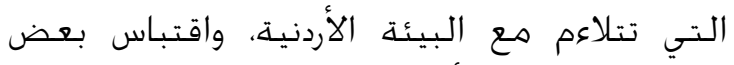

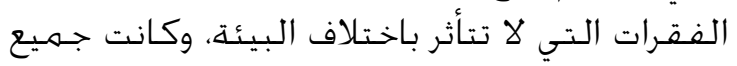
الفقـرات مصـورة.

وقد اقتصــت الدراسـة على اختبار المفردات

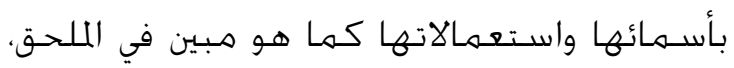

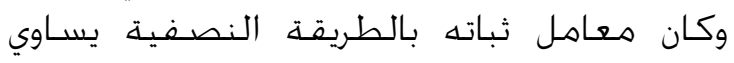

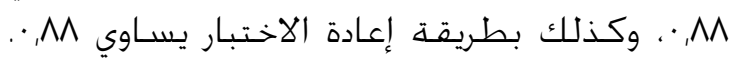

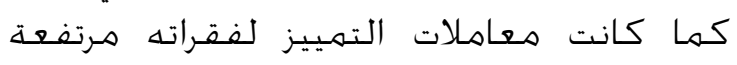

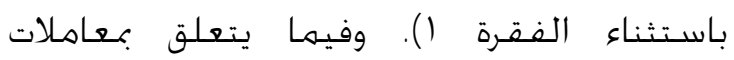

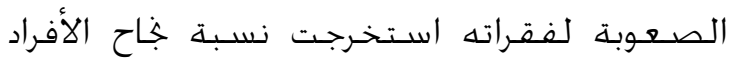

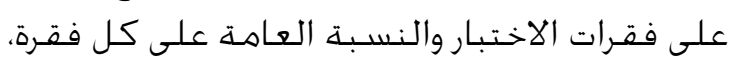

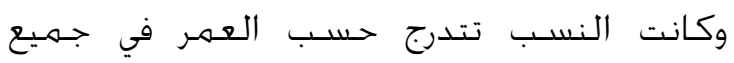




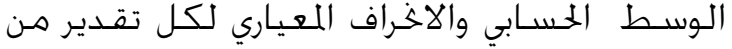

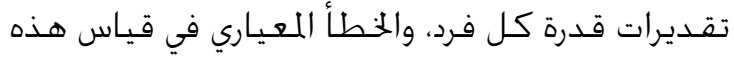

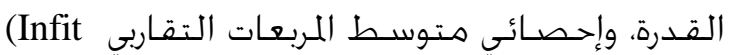
الذي يعبر عن مؤشـر المطابقة الداخلية وإحصائي متوسط مئط المربعات (Infit ZSTD) التباعدي (Outfit MNSQ) الذي يعبر عن مؤشـر (Outit)

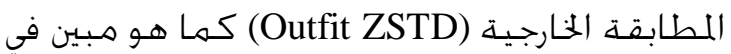

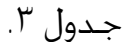

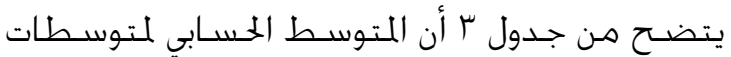

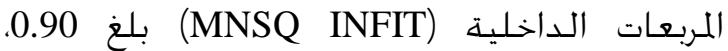
والمتوسط الحسابي لمتوسطات المربعات الخارجية الماتية (MNSQ OUTFIT)

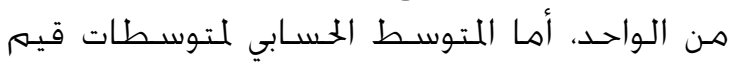

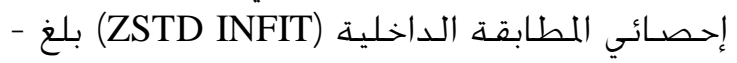

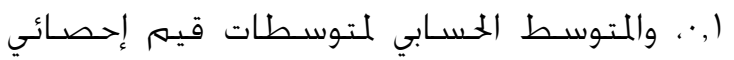

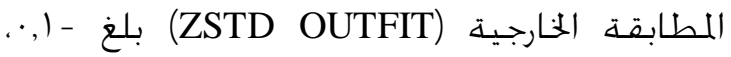

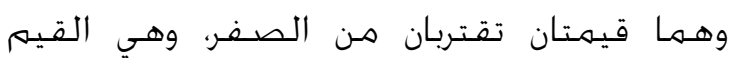

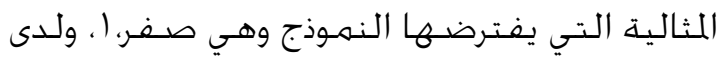

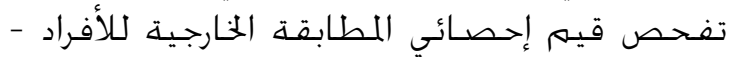

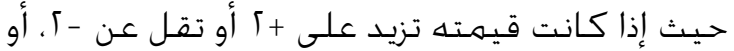

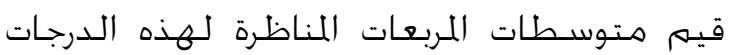
تزيد على ا، وهي القيه التي يتوقعها النمـوذج.

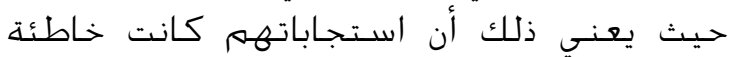

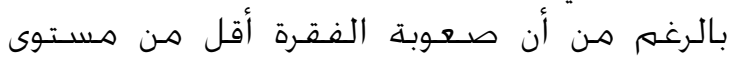

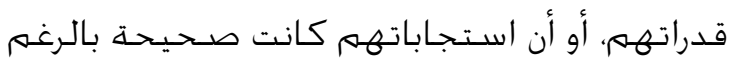
من أن صعوبة الفقرة تفوق مستوى قدراتهم (Wright \& Stone, 1979)

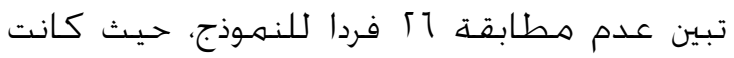
متوسطات المربعات للمطابقة الخارجية لقدراتهم

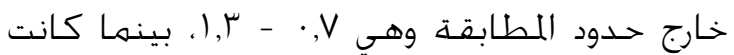

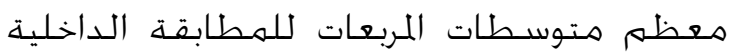

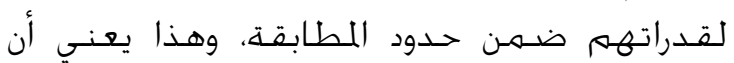

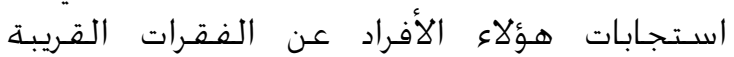

Scree Plot

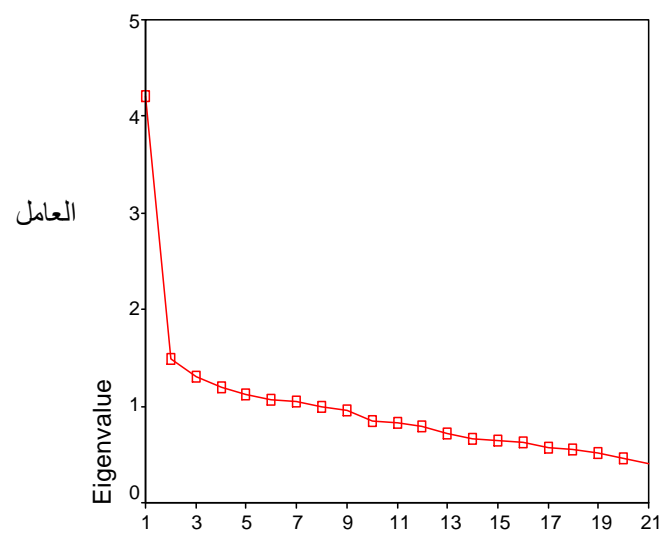

Component Number

شكل1. منحنـى المنحدر لقيم الجذور الكامنة للعوامل اللكونة لاختبار الذكاء.

ونظرا لقلة الفقرات في بعض العوامـل فقد تمت التحل

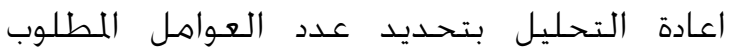

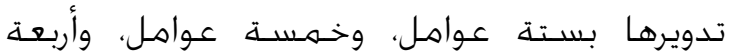

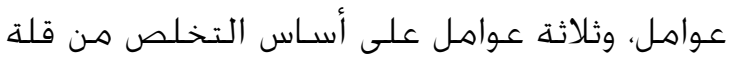

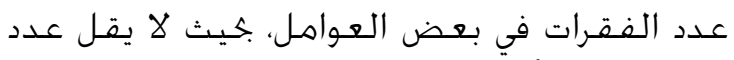

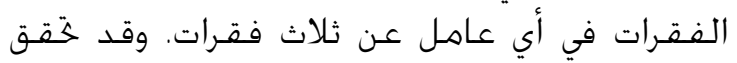

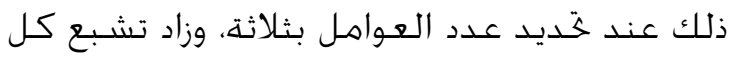

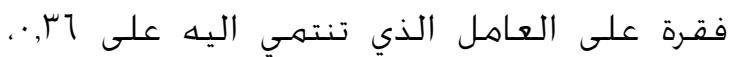

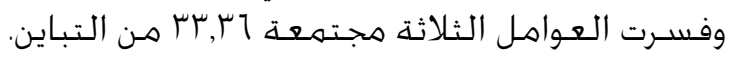
وقد تم استخـدام النمـوذج الثنائي التدريج الذي

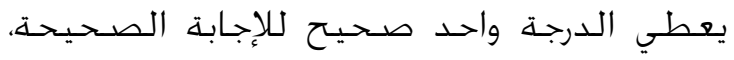

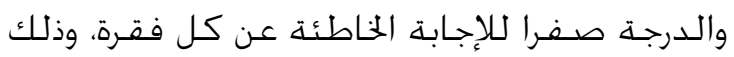

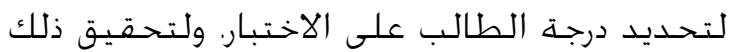

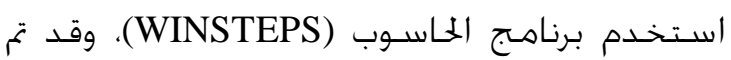

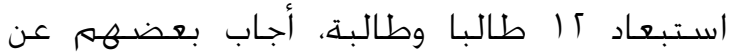

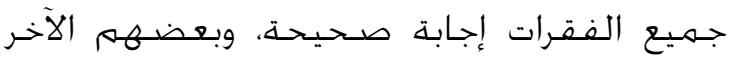

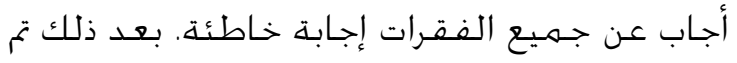

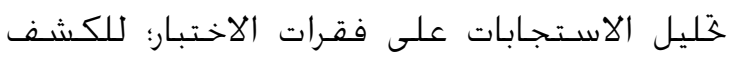
عن مدى مطابقة الأفراد للنموذج، وذلك من الاست خلارل

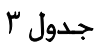

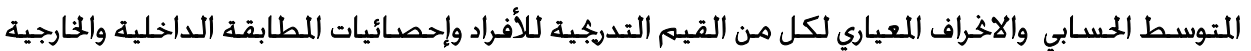

\begin{tabular}{|c|c|c|c|c|c|c|c|}
\hline \multicolumn{2}{|c|}{ إحصائي المطابقة الخارجية } & \multicolumn{2}{|c|}{ إحصائي المطابقة الداخلية } & \multirow[b]{2}{*}{ المعياري } & \multirow[b]{2}{*}{ القدرة } & \multirow[b]{2}{*}{ العلامة الخام } & \\
\hline $\begin{array}{c}\text { الإحصاء } \\
\text { ZSTD }\end{array}$ & $\begin{array}{l}\text { المتربعات } \\
\text { MNSQ }\end{array}$ & $\begin{array}{c}\text { الإحصاء } \\
\text { ZSTD }\end{array}$ & $\begin{array}{l}\text { المربعات } \\
\text { MNSQ }\end{array}$ & & & & \\
\hline$\cdot, 1-$ & $\cdot, 90$ & $\cdot, 1-$ & $\cdot, 9$ &,$\wedge 1$ & $\Gamma, \Sigma$. & $\Gamma \cdot, \cdot$ & المتوسط الحسابي \\
\hline$\cdot, \Lambda$ & 1,45 & $\cdot, 1$ & $\cdot, \mu 9$ & , ¿ 5 & $1, \mu^{5}$ & $\Gamma, \Gamma$ & الاخراف المعياري \\
\hline
\end{tabular}




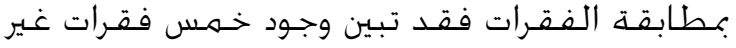

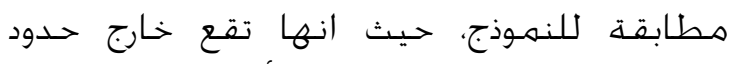

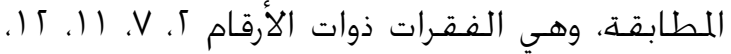

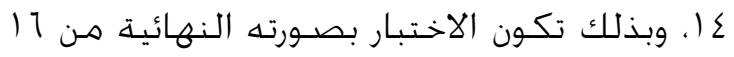

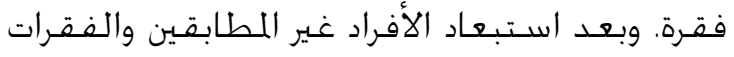

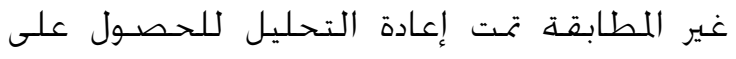

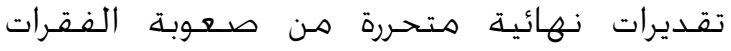

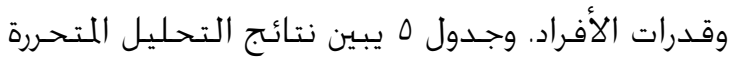
مـن قدرات الأفراد.

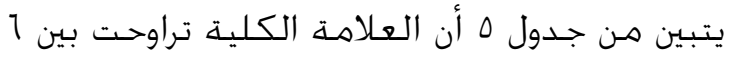

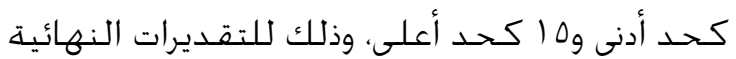

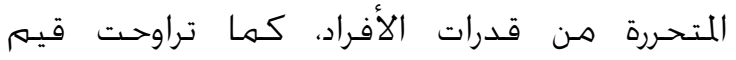

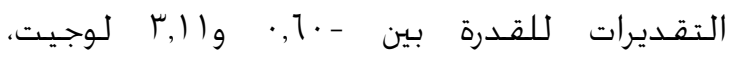

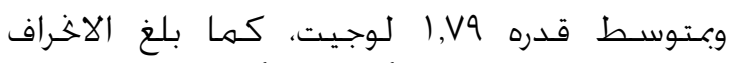

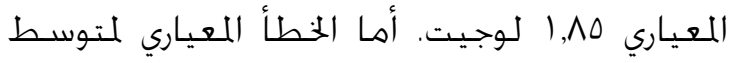

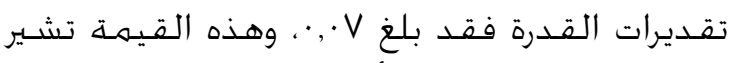

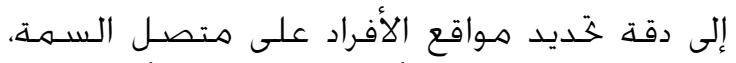

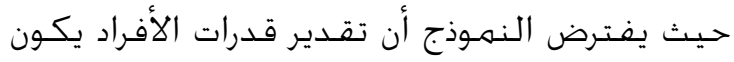

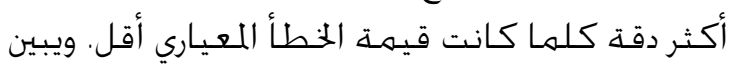

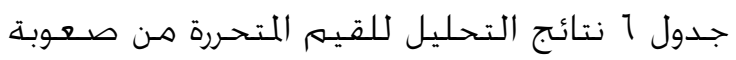

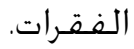

هن هستوى قدراتهم كانت مقبولة، الا أن مقان

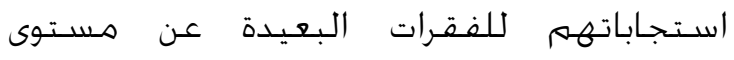

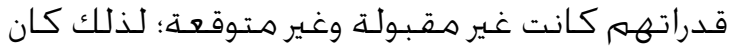
هؤلاء الأفراد غير مطابقين للنمـوذج.

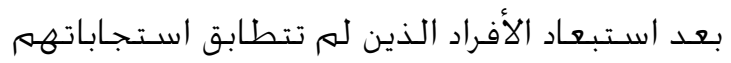

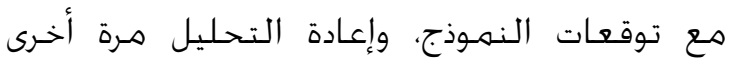

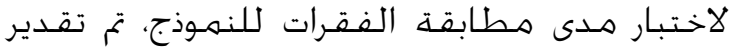

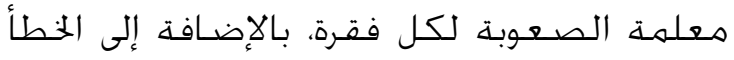

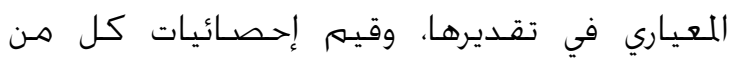
المطابقة الداخلية ارية (Infit ZSTD)، والمطابقة الخارجية (Outfit ZSTD)، وكذلك قيم إحصائيات

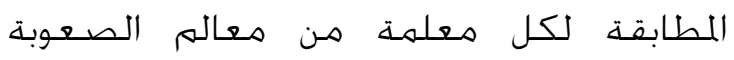

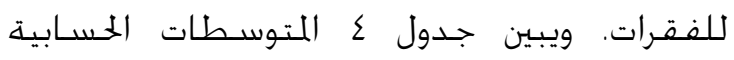

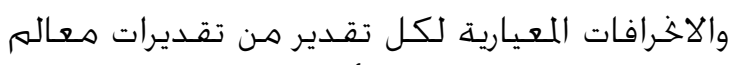

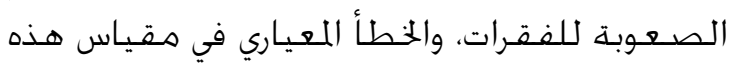

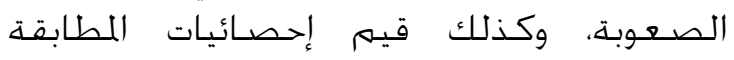
الداخلية والخارجية (MNSQ) لهذهة المعاله.

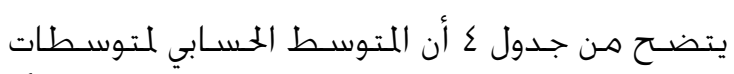

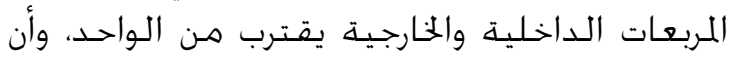

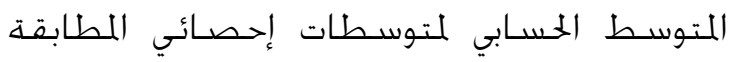

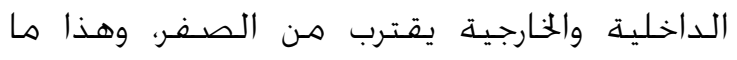

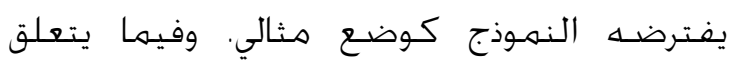

جدول

المتوسط الحسابي والاخراف المعياري لكل من معالم الصعوبة للفقرات والخطأ المعياري، وإحصائيات المطابقة الداخلية والخارجية لهذه المعالم لـفرات

\begin{tabular}{|c|c|c|c|c|c|c|c|}
\hline \multicolumn{2}{|c|}{ إحصلئي المطابقـة الخارجيـة } & \multicolumn{2}{|c|}{ إحصـائي المطابقـة الداخلية } & \multirow{2}{*}{ المعياري } & \multirow[b]{2}{*}{ القــــ } & \multirow[b]{2}{*}{ العلامة الخام } & \\
\hline الإحصـــ & متـوسـط المربعات & الإحســـ & مترسـط المربعات & & & & \\
\hline$\cdot, 1-$ & $\cdot, 9 \mathrm{~V}$ & $\cdot, \cdot$ & $\cdot, 99$ & $\cdot, \Gamma 0$ & $\cdot, \cdot \cdot$ & $1 \leqslant \mathrm{~V}, 0$ & المتوسـط الخسـابي \\
\hline 1, & $\cdot, r$ & $\cdot, \Sigma$ & $\cdot, \cdot \mathrm{V}$ & $\cdot, \cdot V$ & $\cdot, 91$ & $\Gamma \cdot, \cdot$ & الاخـراف المعياري \\
\hline
\end{tabular}

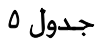

نتائج التحليل للقيم المتحررة من قدرات الأفراد

\begin{tabular}{|c|c|c|c|c|c|c|c|}
\hline \multicolumn{2}{|c|}{ إحصسائي المطابقـة الخارجية } & \multicolumn{2}{|c|}{ إحصـائي المطابقـة الداخلية } & \multirow{2}{*}{ اللعياري الخطأ } & \multirow[b]{2}{*}{ القـدرة } & \multirow{2}{*}{ العلامة الخام } & \\
\hline الإحســاء & متوسط المربعات & الإحســاء & متوسـط المربعات & & & & \\
\hline$\cdot, 1-$ & $\cdot, 9 \leq$ & $\cdot, \cdot$ & $1, \cdots$ & $\cdot, \mathrm{V} \varepsilon$ & $1, \mathrm{V9}$ & $1 \Gamma, \Lambda$ & 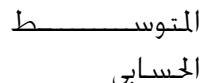 \\
\hline$\cdot, \mathrm{V}$ & $\cdot, \Sigma 1$ & $\cdot, 1$ & $\cdot, \Gamma 1$ & $\cdot, I V$ & $1, \wedge 0$ & 1,9 & الاخراف" المعياري \\
\hline$\ulcorner, \Lambda$ & $r, \Delta \varepsilon$ & $\Gamma, \Sigma$ & 1,00 & $1, \cdot V$ & $r, 11$ & 10 & أعلى علامة \\
\hline $1, \mu-$ & $\cdot, \Gamma 1$ & $\cdot, 1 \varepsilon$ & $\cdot, 1 \varepsilon$ & $\cdot, \Delta \Delta$ & $\cdot, 7 \cdot-$ & 1, & أدنى علامة \\
\hline
\end{tabular}




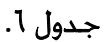

نتائج التحليل للقيم المتحررة من صعوبة الفقرات

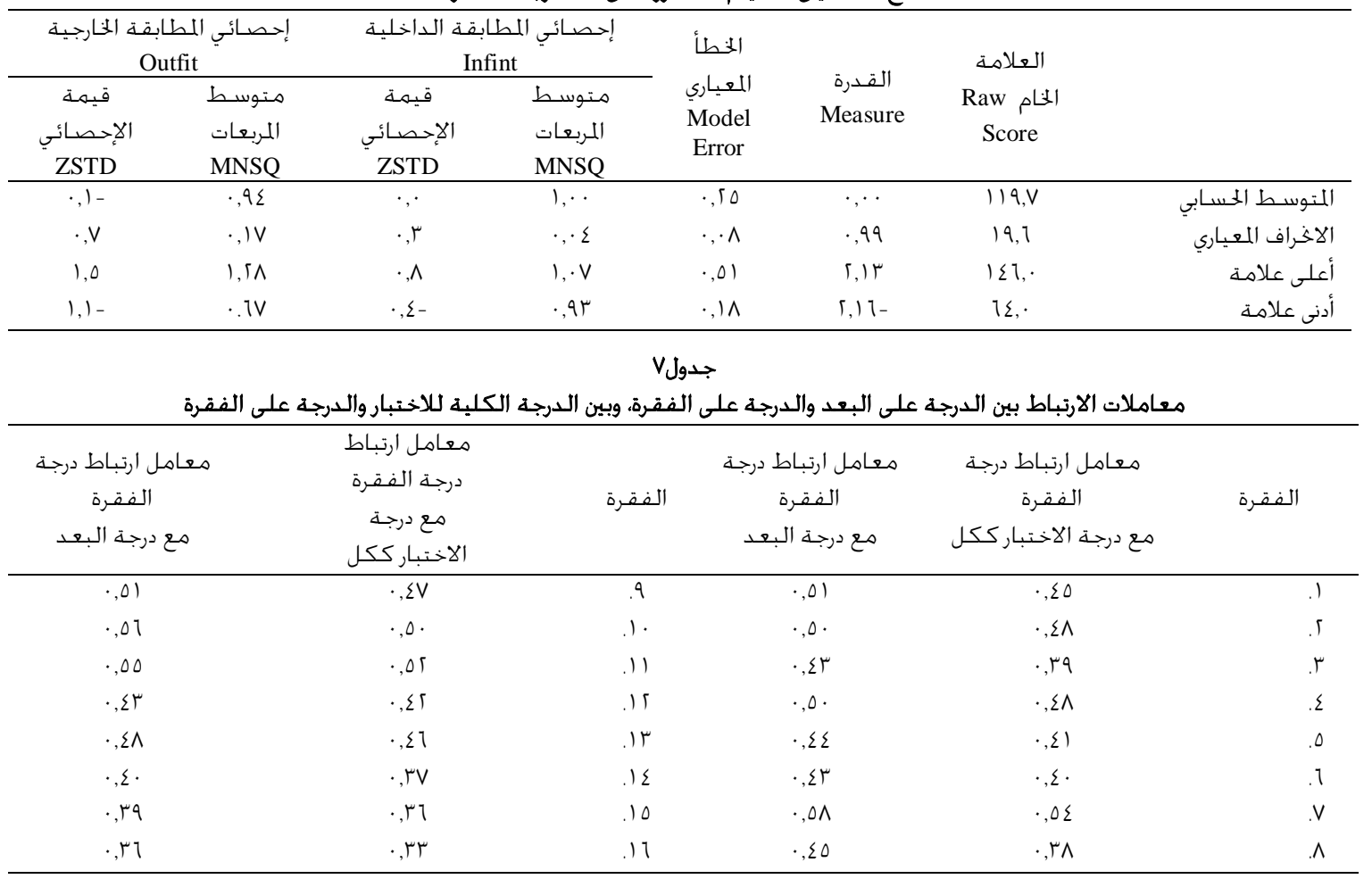

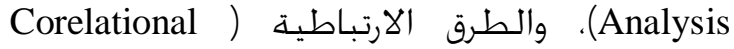
Experimental (Techniques (Techniques

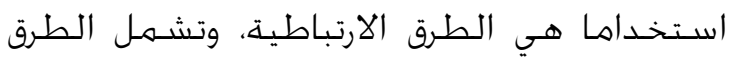

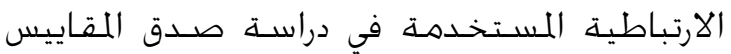

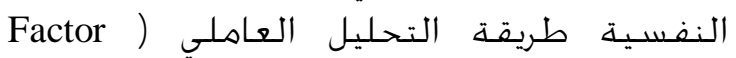

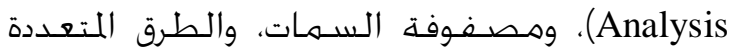
(Multitraite-Multimethod) (Regression Analyses)

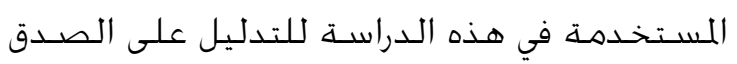

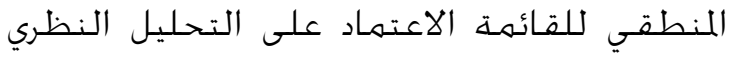

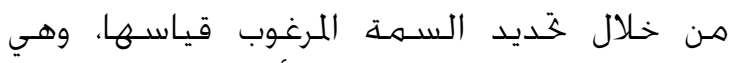

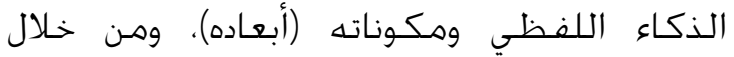

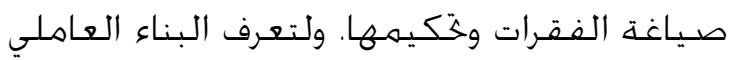

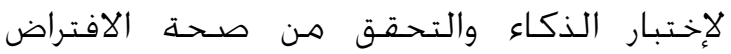

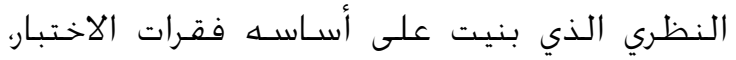

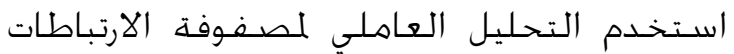

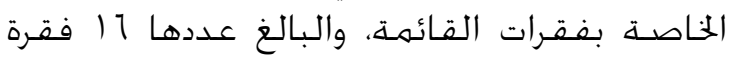
على أفراد العينة الكلية. وجدول V يبين معاملات

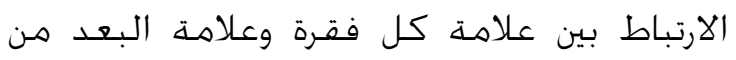

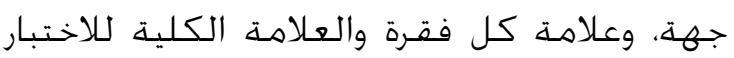
من جهة أخرى.
يشير جدول 1 إلى أن قيم صعوبة الفقـرات تراوحت

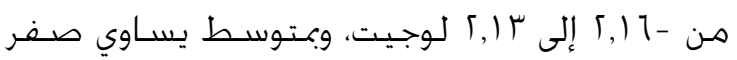
لوجيت، والاخراف المعياري 99,، لوجيت. أما قيمة فيمة

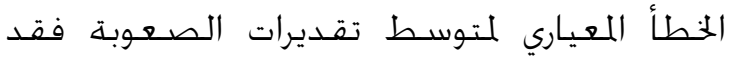
بلغت 1/,· وهي قيمة تشير إلى دقة تقديرات

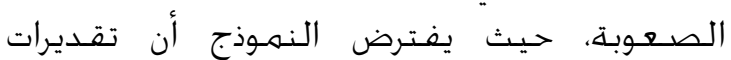

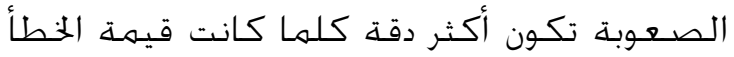
المعياري في قياسـها أقل.

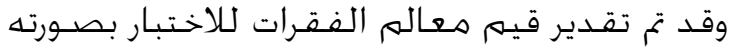

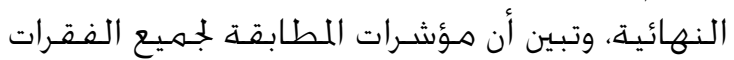
كانت ضـمـن حدود المطابقة الداخلية والخارجية.

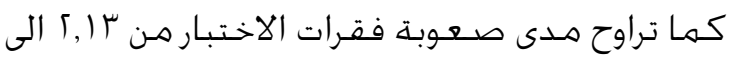

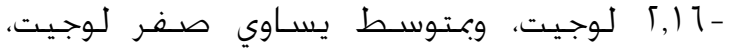

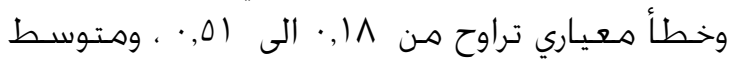

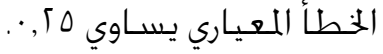

\section{دلالات الصـدق والثبات للاختبار}

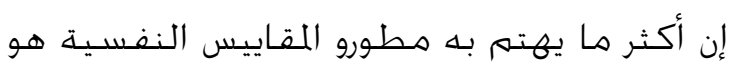

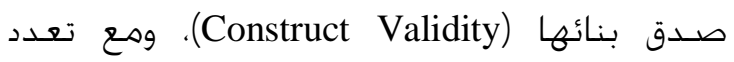

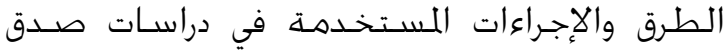

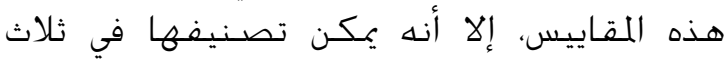
مجهوعات رئيسـة هي: التحليل المنطقي ( Logical 
كمها تم التحقق من دلالات ثبات الاتسـاق الداخلي

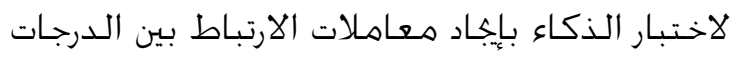

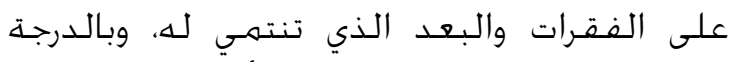

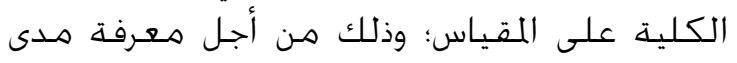

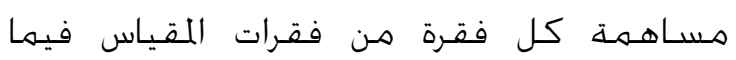

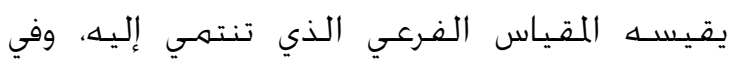

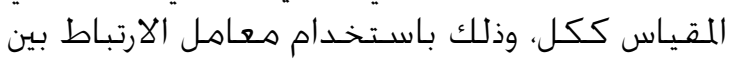

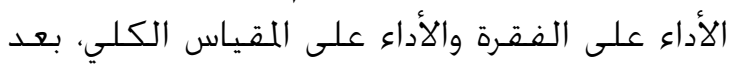

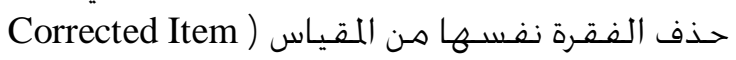

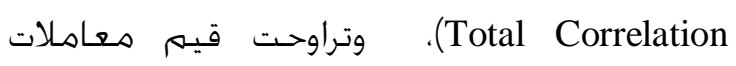

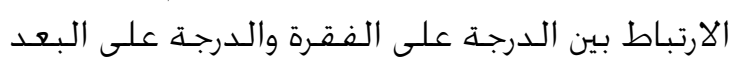

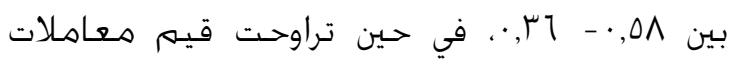

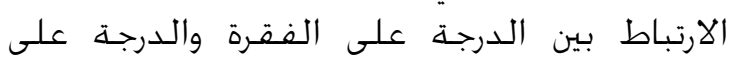

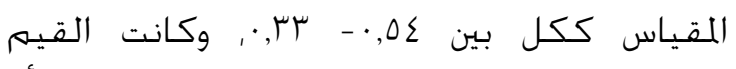

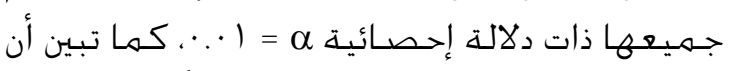

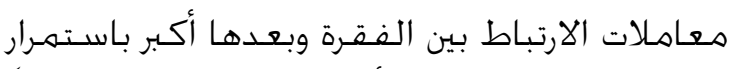
من معامل ارتباطها بالأداة ككل. ويوفر ذلك دلئل ديلاً

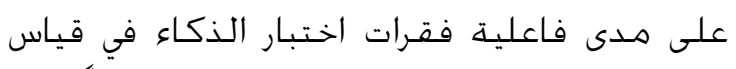

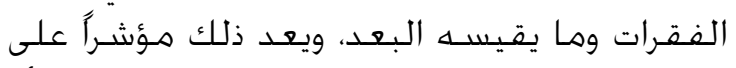

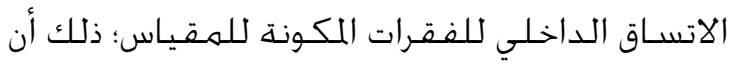

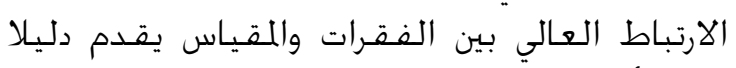

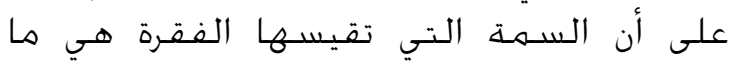

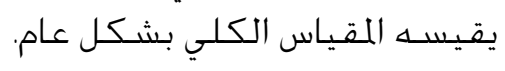

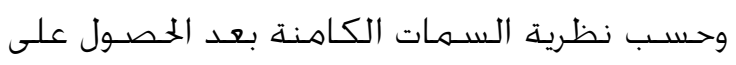

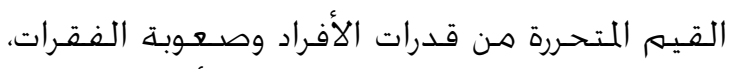

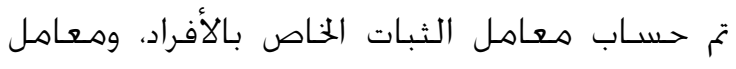

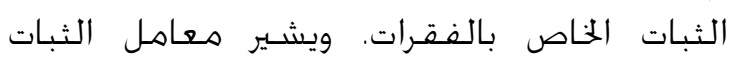

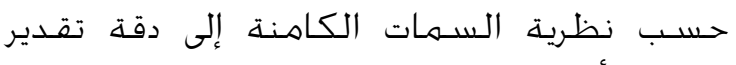

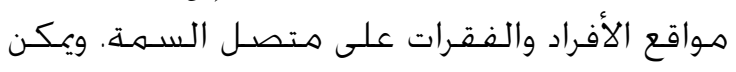

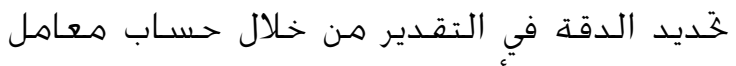
الفصل بين الأفراد (Gerson Separation (Gp) وقد بلغت قيمته 2.05، ومعامل الفصل (Gndex) بين الفقـرات (Gi) (Item Separation Index) وقد بلغت قيمته 3.36 بعد ذلك تم التهرات حساب معامل الثبات للفقـرات والأفراد باستخــــام المعادلة التالية

:(Wright \& Masters, 1982)

$$
R=\frac{G^{2}}{1+G^{2}}
$$

حيث (G) تشـير إلى معـامل الفصـل، (R) إلى معامـل

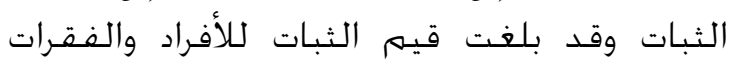

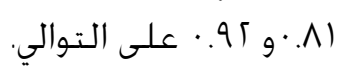

يتبين من جـدول V أن جميع الفقـرات يزيد معامل

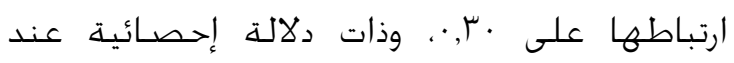
مستوى الدلالة | .,.·، وهذا يدل على على أن الفقـرات 17

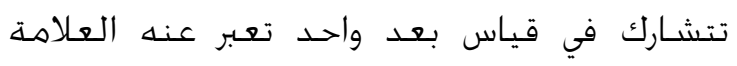

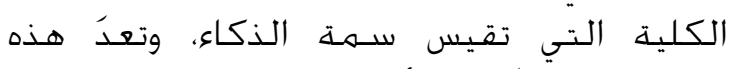

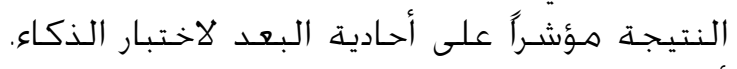

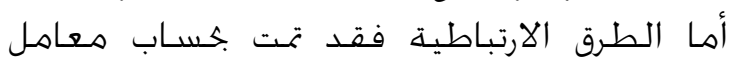

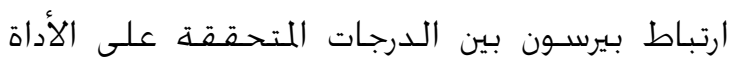

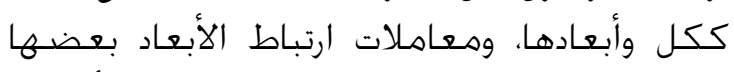

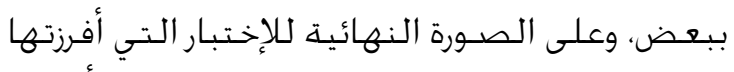

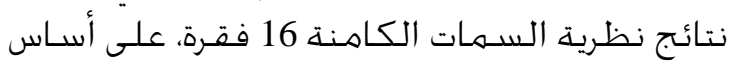

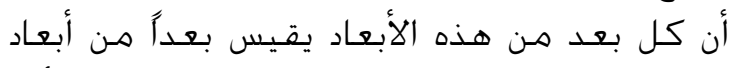

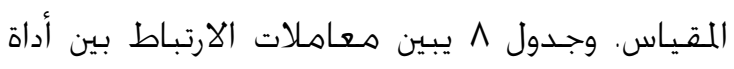

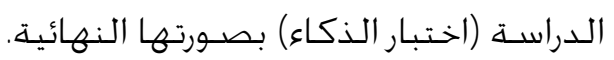

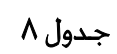
معاملات الارتباط بين الأبعاد الفرعية لاختبار

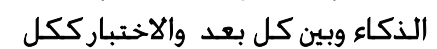

\begin{tabular}{|c|c|c|c|}
\hline الكلي & الثاني & الأول & البعد \\
\hline 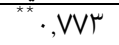 &., 10. & & الأول \\
\hline${ }^{* *} \cdot, V \leq r$ & & & الثاني \\
\hline
\end{tabular}

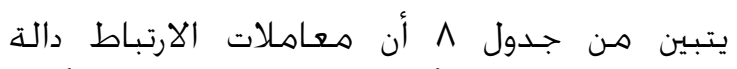

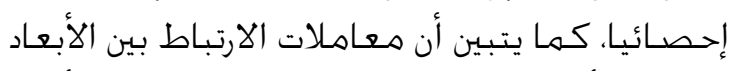

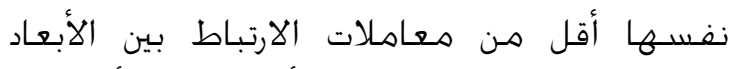
والمقياس الكلي. وهعنى ذلك أنه لا لميكن أن تكون الارنيال

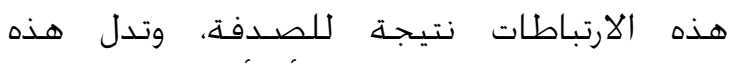
الارتباطات بين الدرجات على أن أبعاد الاختبار غير هذير

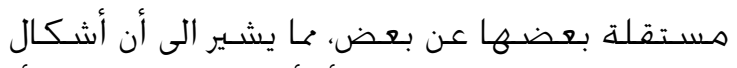

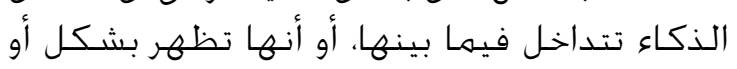

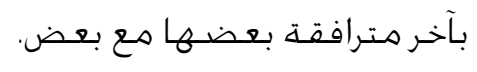
وتم حسـاب معاملات الارتباط بين فقـرات الاختبار

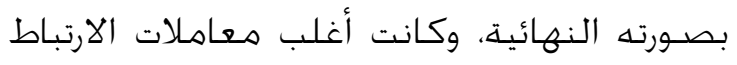

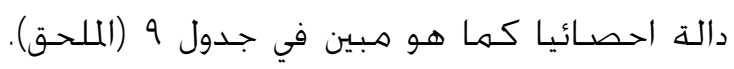

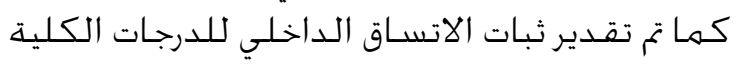

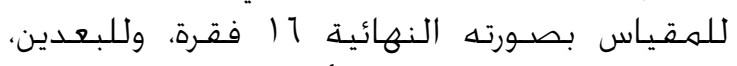

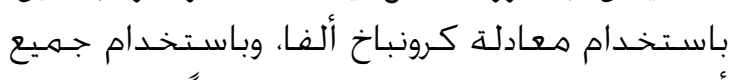

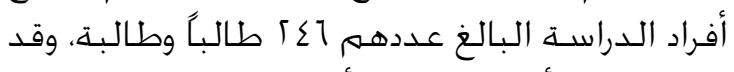

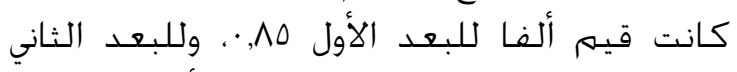
AV

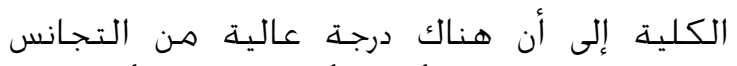

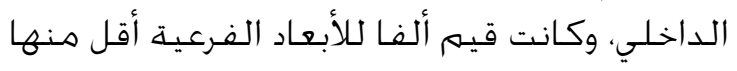
للعلامة الكلية. 
باستخدام نموذج راش (رسـالة ماجستير غير

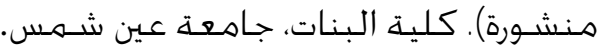

عطوف، محمود ياسين (191)). اختبارات الذكاء

والقدرات العقلية بين التطرف والاعتدال.

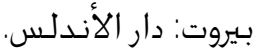

عطية، نعيم (ب94). ذكاء الأطفال من خلال

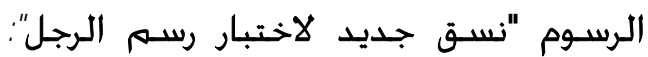
دراسـة تجريبية. بيروت: دار الطليعة.

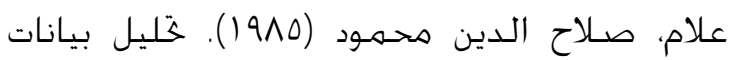

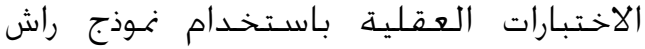

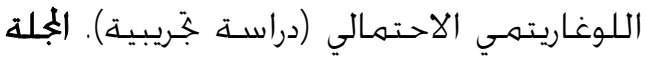

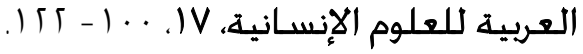

فتحي، هشام (1999). تطوير اختبار كاتل باستخدام نماذج نظرية السمات الكامنة وأثر

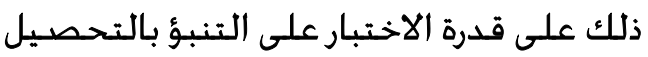

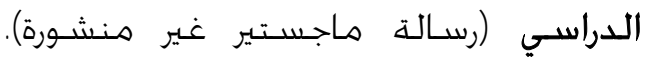
جامعة المنصورة.

هسـعود، وليد (ع + ؟). دراسـة سيكومترية لتطوير

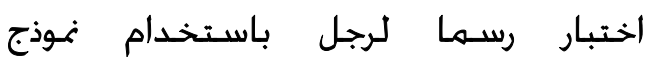

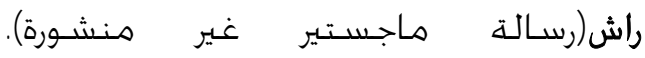
جامعةعينشـمس.

ملكاوي، سـهام عبد اللهّ (9V9 (). تطوير اختبار ذكاء

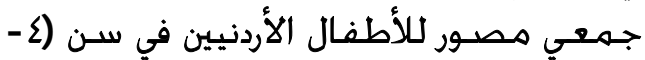
9) سنوات (رسـالة ماجستير غير منشـورة). الجامعة الأردنية.

Ackerman, P. L., \& Kanfer, R. (2004). Cognitive, affective, and conative aspects of adult intellect within a typical and maximal performance framework. In D.Y.Dai \& R.J. Sternberg (Eds), M otivation, emotion, and congnition: Integrated perspectives on intellectual functioning ( $p p$. 119-141). Mahwah, NJ: Erlbaum.

Acton, G. S. (2003). What is good about Rasch measurement? Rash M easurement Transaction, 16, 902- 903.

Aiken, J. R. (2003). Psychlogicaltesting and assessment. (11 $1^{\text {thed. }}$.). Boston: Person Education Group, Inc.

Albert, J. H., \& Ghosh, M. (2000). Item response Modeling. In: D. Day. S. Gohst and Mallick.
الخلاصـة والتوصيات

هدفت هذه الدراسـة إلى تقصي الخصائص

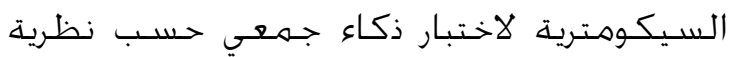
السـمات الكامنه وقد تكون الاختبار بصـورته الائه

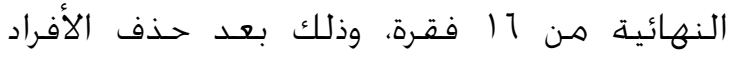

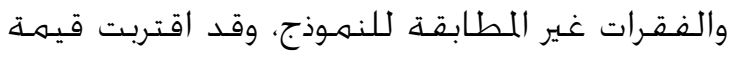

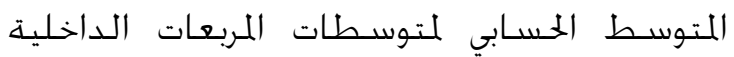

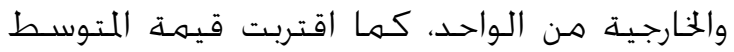

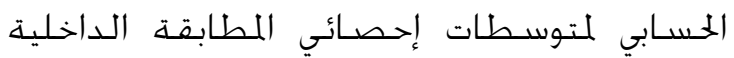

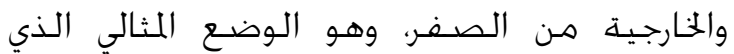

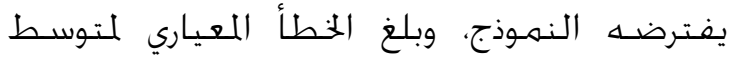

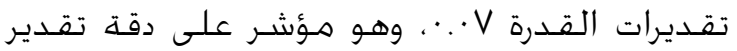

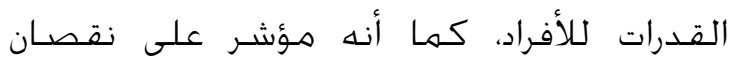

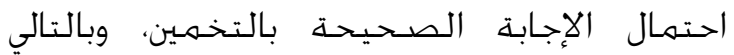

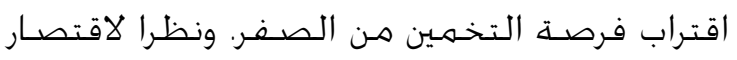

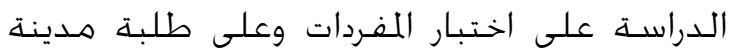

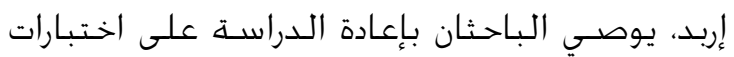

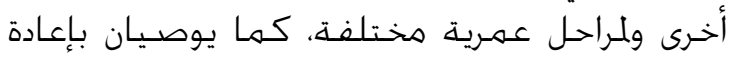

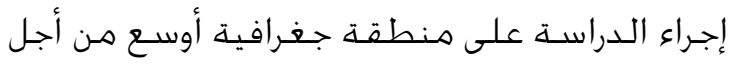
تعزيز الثقة بالخصائص السيكومترية للاختبار.

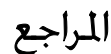

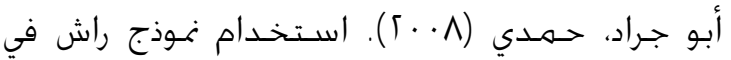

تطوير اختبار كاتل الثالث للذكاء الصـاء الصـورة (أ).)

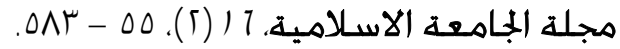

حسـان، شفيق (.99 (). العلاقة بين بعض العواهل

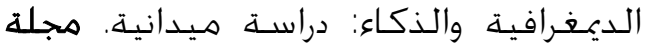

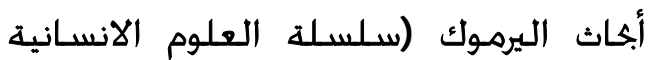

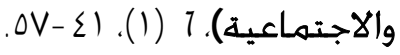

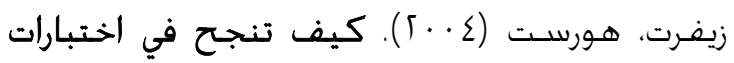

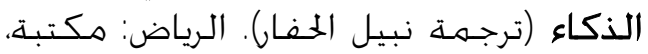

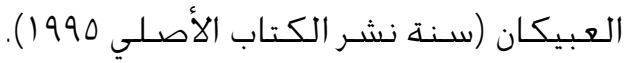
الطريري، عبدالرحمن (1997). الخصائص

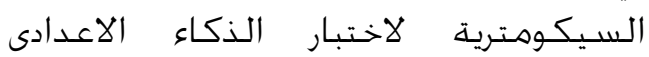

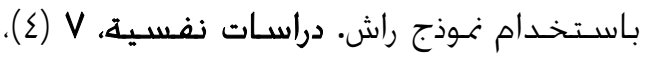
. $\Sigma V^{\mu}-\Sigma O V$

الطنطاوي، منـى ( ( . ؟). دراسـة سيكومترية حول

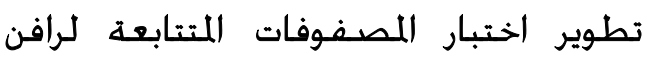


(Eds.). Generalized linear models: A Bayesian perspective (pp. 173-193). NewYork: Marcel- Dekker.

Anastasi, A. (1982). Psychological testing ( $5^{\text {thed. }}$.). New York: Macmillan Publishing Co.

Anastasi, A., \& Urbina, S. (1997). Psychological testing. ( $7^{\text {th }}$ ed.). New Jersey: Prentic-Hall, Inc.

Anderson, J. (1990). Cognitive P sychology and its Implications ( $3^{\text {rd }}$ ed.). New York: W. H. Freeman and Company.

Collins, J. \& Messick, S. (2001). Intelligence and personality: Bridging the gap in theory and measurement. Hillsdale NJ: Erlbaum.

Crocker, L., \& Algina, J. (1986). Introduction to classical and modern test theory. New York: CBSCollege Publishing.

Cronbach, L. (1960). Essential of psychological testing. New York: Harper and Bros.

Cronbach, L. (1971). Test validation. In R. L. Thorndike (Ed.), Educational measurement (2nd ed., pp. 443-507). Washington, DC: American Council on Education.

Curtis, D. (2001). Misfit: People and their problems, what might it all mean? International Education Journal, 2(4), 91-99.

El-Korashy, A. (1995). Applying the Rasch model to the selection of items for a mental ability test. Educational and Psychological M easurement, 55(5), 753-763.

Eysneck, H. (1997). Personality and experimental psychology: the unification of psychology and possibility of a paradigm. Journal of Personality and Social Psychology, 73, 1224-1237.

Fan, X.(1998). Item response theory and classical test theory: an empirical comparison of their item/ person statistics. Educational and Psychological M easurement, 58 (3), 357-373.
Gottfredson, L., \& Saklofske, D. H. (2009). Intelligences: foundations and issues in assessment. Canadian Psychology, 50 (30), 183- 195.

Hambleton, R. K., and Swaminathan, H. (1985). Item response theory principles and applications. Boston: Kluwer, Nijhoff Publishing.

Hashmi, M. A., Tirmizi, S.H., \& Shah, A.F. (2010). Development and validation of intelligence test for grade six students (Age group 11-12 years). Journal of Educational Research, 13(2), 19-29.

Hattie, J. (1985). Methodology review: Assessing unidimensionality of tests and items. A pplied Psychological M easurement, 9, 139-164.

Herrnstein, R. J., \& Murray, C. (1994). The Bell Curve: Intelligence and Class Structure in A merican Life New York: Free press.

Loyd, R. (2004). Emotional intelligence and stress coping in dental undergraduates, a qualitative study. Educational Psychological Research, 197(4), 209-265.

Masters, G. N. (1982). A Rasch model for partial credit scoring. Psychometrika, 2, 149174.

Touloumtzoglou, J. (1999). Pilot study of the visual arts attitude scale (from $A$ and from B). Retrieved October 18, 2011 from: http://ehtl.flinders.edu.au/education/publicatio ns/JOURN A LS/touloumtzoglou999/BEGIN.

Wright, B. D., \& Master, G.N. (1982). Rating scale analysis. Rasch measurement. Chicago: MESA Press.

Wright, B. D., \& Stone, M. (1979). Best test design. A handbook for Rasch measurement. Chicago: MESA Press. 
ملحق 1

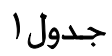

قيم معاملات الارتباط بين فقرات الاختبار

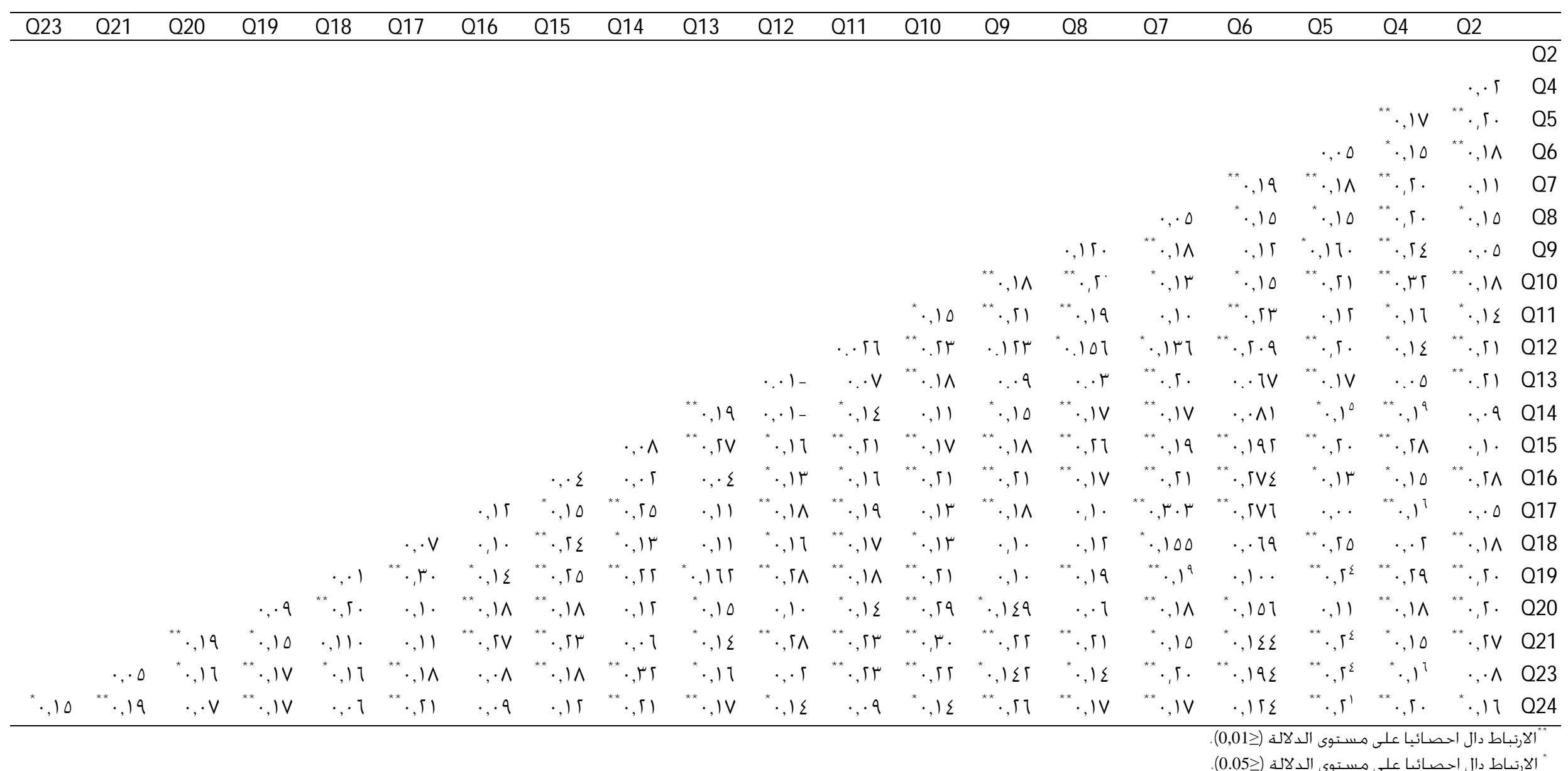




\begin{tabular}{|c|c|c|c|c|c|c|c|c|c|c|c|c|c|c|c|c|}
\hline \multicolumn{17}{|c|}{ قبم معاملات الارتباط بين فقرات الاختبار بصورته النهائية } \\
\hline Q21 & Q20 & Q19 & Q18 & Q17 & Q16 & Q15 & Q14 & Q13 & Q10 & Q9 & Q8 & Q6 & Q5 & Q4 & $\overline{Q 2}$ & \\
\hline & & & & & & & & & & & & & & & $\cdot, \cdot 10$ & Q4 \\
\hline & & & & & & & & & & & & & & ${ }^{* *} \cdot, I V \Gamma$ & ${ }^{* *} \cdot, 19 \wedge$ & Q5 \\
\hline & & & & & & & & & & & & & $\cdot, \cdot \Sigma 9$ & ${ }^{*} \cdot, 1 \leq 9$ & ${ }^{* *} \cdot, I V V$ & Q6 \\
\hline & & & & & & & & & & & & ${ }^{*} \cdot, 1 \Delta r^{\prime}$ & ${ }^{*} \cdot, 1 \leq 0$ & ${ }^{* *} \cdot, 19 \wedge$ & ${ }^{*} \cdot, 10$ & Q8 \\
\hline & & & & & & & & & & & . & $\cdot, 110$ & ${ }^{*} \cdot, 17$ & ${ }^{* *} \cdot, \Gamma \leq \Gamma$ & $\cdot .05$ & Q9 \\
\hline & & & & & & & & & & ${ }^{* *} \cdot,|\wedge|$ & ${ }^{* *} \cdot, 190$ & ${ }^{*} \cdot, 1 \leqslant \Lambda$ & ${ }^{* *} \cdot, \Gamma 1 \%$ & ${ }^{* *} \cdot, \mu \Gamma \mu$ & ${ }^{* *} \cdot, \| \wedge r$ & Q10 \\
\hline & & & & & & & & & ${ }^{* *} \cdot, \mid \wedge \Gamma$ & $\cdot, M \Lambda$ & $\cdot, \cdot 19$ & $\cdot, \cdot \mathrm{TV}$ & ${ }^{* *} \cdot,|\mathrm{V}|$ & $\cdot, .0 T$ & $\left.{ }^{* *} \cdot, \Gamma\right\rceil$ & Q13 \\
\hline & & & & & & & & ${ }^{* *} \cdot, 19$ & $\cdot, 1 \cdot 0$ & ${ }^{*} \cdot, 10$ & ${ }^{* *} \cdot,|,| \mathrm{V}$ & $\cdot, \cdot \wedge l$ & ${ }^{*} \cdot, 1 \leq 9$ & ${ }^{* *} \cdot, 1 \wedge \Delta$ & $\therefore \wedge 19$ & Q14 \\
\hline & & & & & & & $\cdot, \cdot V q$ & ${ }^{* *} \cdot, \Gamma 1 \varepsilon$ & ${ }^{* *} \cdot, I V \Sigma$ & ${ }^{* *} \cdot, \mathrm{IV \Lambda}$ & ${ }^{* *} \cdot$, Г Г & ${ }^{* *} \cdot, 195$ & $\left.{ }^{* *} \cdot, \Gamma \cdot\right)$ & ${ }^{* *} \cdot,\lceil\Lambda$ & $\cdot, .99$ & Q15 \\
\hline & & & & & & $\cdot, \cdot \sum r$ & $\cdot, \cdot 11$ & $\cdot, \cdot r V$ & $\left.{ }^{* *} \cdot, \Gamma\right) \Gamma$ & ${ }^{* *} \cdot, \Gamma \cdot V$ & ${ }^{* *} \cdot, 179$ & ${ }^{* *} \cdot, \Gamma V \varepsilon$ & ז & ${ }^{*} \cdot, 1 \leq 0$ & ${ }^{* *} \cdot$, IVq & Q16 \\
\hline & & & & & $\cdot, 117$ & ${ }^{*} \cdot, 1 \Delta r^{\prime}$ & ${ }^{* *} \cdot, \Gamma \Delta r$ &., 111 & $\cdot, 11 \pi$ & ${ }^{* *} \cdot,|\wedge|$ & $\cdot, \cdot 90$ & ${ }^{* *} \cdot$, 「V 1 & $\cdot, \cdots$ & ${ }^{* *} \cdot, 190$ & $\cdot, \cdot 01$ & Q17 \\
\hline & & & & $\cdot, \cdot V_{1}$ & $\cdot, 9 \mathrm{~V}$ & ${ }^{* *} \cdot$, гाq & ${ }^{*} \cdot, 1 \Gamma 0$ & $\cdot, 111$ & ${ }^{*} \cdot, \mu \mu \mu$ & $\cdot, 90$ &., 117 & $\cdot, \cdot 79$ & ${ }^{* *} \cdot$, го & $\cdot, \cdot \Gamma$ & ${ }^{* *} \cdot,, \wedge$. & Q18 \\
\hline & & & $\cdot, \cdot 1 \varepsilon$ & ${ }^{* *} \cdot,\lceil 91$ & ד] & $\left.{ }^{* *} \cdot, \Gamma \Delta\right)$ & ${ }^{* *} \cdot$, Гा & ${ }^{*} \cdot, 171$ & $\left.{ }^{* *} \cdot, \Gamma\right)$ & ., I. & ${ }^{* *} \cdot, 191$ & $\cdot, 1 \ldots$ & ${ }^{* *} \cdot, \Gamma \mathrm{V}$ & ${ }^{* *} \cdot, \Gamma 9 \varepsilon$ & ${ }^{* *} \cdot, 190$ & Q19 \\
\hline & & $.9 \varepsilon$ & ${ }^{* *} \cdot, \Gamma \cdot \Gamma$ & $\cdot, 1.5$ & ${ }^{* *} ., I V \wedge$ & ${ }^{* *} \cdot, 1 \wedge \mu$ &., 110 & ${ }^{*} \cdot, 10$ & ${ }^{* *} ., \Gamma q \mu$ & ${ }^{*} \cdot, 1 \leq 9$ & ז., & ${ }^{*} ., 107$ &., $11 \varepsilon$ & ${ }^{* *} \cdot, 1 \wedge \Sigma$ & ${ }^{* *} \cdot, 199$ & Q20 \\
\hline & ${ }^{* *} \cdot,, M \Lambda$ & ${ }^{*} \cdot, 1 \leq 0$ & $\cdot, 11$ & $\cdot, 11$. & ${ }^{* *} \cdot, \Gamma \mathrm{V}^{\mu}$ & ${ }^{* *} \cdot, \Gamma \mu \mu$ & $\cdot, \cdot 7 \mu$ & ${ }^{*} \cdot, 1 \% q$ & ${ }^{* *} \cdot, \Gamma 99$ & ${ }^{* *} \cdot, \Gamma \Gamma$. & ${ }^{* *} \cdot, \Gamma$. & ${ }^{*} \cdot, 1 \leq \varepsilon$ & ${ }^{* *} \cdot, \Gamma \mu$ ] & ${ }^{*} \cdot, 105$ & $\left.{ }^{* *} ., \Gamma\right\rceil \wedge$ & Q21 \\
\hline
\end{tabular}

